\title{
Elmar Allivater
}

\section{Die Ordnung rationaler Weltbeherrschung oder: Ein Wetthewerb von Zauberlehrlingen}

Von einer »Weltordnung « kann man, wenn überhaupt, erst im 20. Jahrhundert sprechen. Die »globalen Ordnungen« der Jahrhunderte zuvor umfaßten immer nur die jeweils bekannte und erreichbare, niemals aber die ganze Welt. Wenn sie denn Ordnungen, also dauerhaft und als politischer Entwurf angelegt waren, balancierten sie die Kräfte souveräner Nationalstaaten, wie in der »pax westphalica« nach dem 30jährigen Krieg, deren Grundprinzip bis ins 20. Jahrhundert nachwirkte (Miller 1994). Doch zu viele Elemente waren in einem System von Nationalstaaten zu regulieren, das wie nach den Newton'schen Gesetzen der Schwerkraft ein »Gleichgewicht der Kräfte « hervorbringen sollte. Es kam unweigerlich ins Trudeln, als die Übersichtlichkeit des Systems verloren ging und zu viele Elemente von zu unterschiedlichen Gewichten und Interessen im Gleichgewicht zu halten waren, zumal Nationalstaaten, deren Souveränität in der Fähigkeit besteht, Grenzen zu setzen, im Zeitalter des Imperialismus danach strebien, die Welt zu erobern, indem sie die nationalstaatlichen Grenzen ausdehnten. Es ist leicht auszurechnen, daß dies zum Konflikt führt, wenn auch nur zwei Nationalstaaten dem gleichen Prinzip folgen. Die beiden Weltkriege dieses Jahrhunderts beendeten die Ära der Ordnung von Nationalstaaten.

\section{Von der Weltordnung von Nationalstiaten zu einer Welt von "Wethewer最sstaater"}

Das Hauptcharakteristikum der bipolaren Weltordnung der zweiten Hälfte des 20. Jahrhunderts, von der Erarbeitung des »grand design« der Nachkriegszeit bis zum Fall der Mauer 1989 ist der »Systemwettbewerb zwischen Staatenblöcken, zwischen kapitalistischem Westen und real-sozialistischem Lager. Die noch im 19. Jahrhundert einheitliche Geschichte gerät 1917 an eine Bifurkation, von der damals und auch 1947, als der »kalte Krieg « begann, nicht zu sagen war, welche Verzweigung eher »der $\mathbb{Z} u-$ kunft zugewandt « sein sollte. Beide »historischen $\ll$ Systeme ${ }^{1}$ beanspruch-

1 Immanuel Wallerstein hat für die kapitalistische Produktionsweise die historischen Ligaturen herausgearbeitet und neben die systematische Analyse die historische Verortung gestellt (Wallerstein 1984). 
ten, der Welt eine (jeweils alternative) Ordnung von Frieden und Entwicklung bieten zu können, wobei im kapitalistischen Westen die individuelle Freiheit, im realsozialistischen Osten die soziale Gleichheit betont werden, und in den nicht festgelegten Weltregionen des »Südens« werben sie beide um Einfluß für ihre Vorstellung von einer Ordnung der Welt. Aber nicht nur zur Austragung des Ost-West-Konflikts ist im Westen ein differenziertes Regelwerk unter der zunächst uneingeschränkten Hegemonie der USA entstanden, das einen brauchbaren Rahmen für Akkumulation in der Zeit und Expansion im Raum geboten hat. Zum Regime der Nachkriegsordnung gehören die Vereinten Nationen, aber auch die globalen Regelsysteme von Handel und Finanzbeziehungen mit den mächtigen, gegen Kriegsende geschaffenen Institutionen von Bretton Woods (Internationaler Währungsfonds und Weltbank) und Havanna (das GATT). Es entstehen regional integrierte Wirtschaftsräume ebenso wie regionale politische Bündnissysteme, die schon deshalb in globalen Zusammenhängen eingebunden sind, weil die Hegemonialmacht USA als »global player « eine Klammer zwischen den regionalen Bündnissen zwischen Atlantik und Pazifik darstellt.

Weltproduktion und Welthandel expandierten mit historisch einmalig hohen Wachstumsraten ${ }^{2}$. Während der langen aufstrebenden Welle der Akkumulation steigt daher die »systemic openess«: der Anteil des Außenhandels am Sozialprodukt wächst (in der OECD von 15,1\% im Jahre 1950 auf 24,8\% im Jahre 1986 - Maddison 1989: 143), die Zollsätze sinken (bei Industriewaren um ca. $60 \%$ in den vergangenen acht Zollrunden), die regionale und nationale Abschottung (Autarkiestreben; Protektionismus) nimmt ab (Krasner 1976, S. $317 f f$; Thompson/Vescera 1992, S. 493ff). Letztere Beobachtung kann nicht ganz unwidersprochen bleiben. Denn die EG wird 1957 gebildet und löst zu Beginn der 60er Jahre eine »erste Welle der Blockbildung « auch in anderen Weltregionen aus (Anderson/Blackhurst 1993; Langhammer 1990). Allerdings hatten diese »regional integration arrangements « (RIA's), obwohl sie mit dem Freihandelsprinzip des GATTAbkommens nicht ohne weiteres kompatibel waren, keine die Welthandelsströme umlenkenden, sondern den Handel insgesamt belebenden Effekte; das Kriterium Viners für die Verträglichkeit einer Zollunion mit dem Freihandelsprinzip wurde also erfüllt (Viner 1950). Daher konnten sie das

2 Das Pro-Kopf-Bruttoinlandsprodukt in 32 Ländern, die von Angus Maddison untersucht worden sind (16 OECD-Länder, 6 lateinamerikanische und 9 asiatische Länder sowie die UdSSR), ist zwischen 1900 und 1950 um 1,1\% im Jahresdurchschnitt gewachsen, zwischen 1950 und 1987 aber um 2,5\%. Das Exportvolumen der 32 Länder nahm von 1900 bis 1950 um jahresdurchschnitlich $1,5 \%$ zu, zwischen 1950 und 1986 aber um jahresdurchschnittlich 6,5\% (Maddison 1989: 14, 26). 
Placet der Freihändler im GATT erhalten. Erst in der »neuen Weltordnung « in den 90er Jahren, als eine »zweite Welle der regionalen Blockbildung « (ebenfalls Anderson/Blackhurst 1993) über den Weltmarkt schwappt, ist dies anders: Mit der wachsenden globalen Konkurrenz wurden auch die Tendenzen der regionalen Abschottung stärker. Im gleichen Maße wie die tarifären Handelshemmnisse in mittlerweile acht GATTRunden abgebaut worden sind, wurden nicht-tarifäre Handelshemmnisse errichtet und regionale »Festungen «, zumal in der Welt der Industrieländer, auf- und ausgebaut, um in der »Triadenkonkurrenz « gewappnet zu sein.

Nationalstaaten hören zwar auf, mit der an Territorium und Staatsvolk gebundenen (nationalstaatlichen) Macht und Souveränität im »Konzert der Mächte《 so wie in der »westfälischen Ordnung《 mitzuspielen. Aber sie behalten bis in die 70er Jahre wichtige souveräne Rechte der Bestimmung zentraler ökonomischer Größen, wie der Wechselkurse und der Zinsen. So ist es möglich, nach dem zweiten Weltkrieg - wie von Keynes theoretisch begründet (Keynes 1936) und von Lord Beveridge (1946) zum politischen Programm erhoben - eine Politik der Vollbeschäftigung und des sozialstaatlichen Ausgleichs (in den Grenzen einer kapitalistischen Ökonomie) zu betreiben. Die internationalen Institutionen sind eher hilfreich bei der Verfolgung der nationalstaatlichen Ziele. Erst nach dem Kollaps des 1944 geschaffenen Weltwährungssystems zu Beginn der 70er Jahre ändert sich dies. Die an das Medium Geld gebundene wirtschaftspolitische Souveränität der Nationalstaaten geht verloren, nachdem sie die territorialräumliche Souveränität teilweise an supranationale Instanzen und an eine Hegemonialmacht haben zedieren müssen. Die internationalen Institutionen der Regulation von Geld, Währung, Finanzen und Handel machen seitdem einen tiefgreifenden Funktionswandel durch. Die Weltordnung der Nachkriegszeit wird bereits in dieser Entwicklungsphase brüchig, nicht erst mit dem Verschwinden des Blockgegners nach 1989. Gleichzeitig versuchen die Nationalstaaten (und Regionen), die verlorene Souveränität über Zinsen, Wechselkurse und Preise auf anderem Terrain, am jeweiligen »Standort《 zurückzugewinnen: durch ein politisches Programm der Herstellung »systemischer Wettbewerbsfähigkeit «3 3 . Die Kosten sollen gesenkt

3 Fritz W. Scharpf hat aus dem Verlust der nationalstaatlichen $»$ Zinssouveränität« die Schlußfolgerung in Richtung eines politischen Konzepts des angebotspolitischen »Sozialismus in einer Klasse« gezogen (Scharpf 1987). Die neuere Debatte um die Wettbewerbsfähigkeit einer weltmarktoffenen Ökonomie betont die regional vernetzten Strukturen von Ökonomie, Gesellschaft, Politik, Kultur als Basis internationaler Wettbewerbsfähigkeit (vgl. Messner/Meyer-Stamer 1993). In dieser Debatte zählen ausschließlich die beeinflussbaren nationalen Bedingungen für Stärke oder Schwäche der Konkurrenzposition, das globale System und seine Funktionsweise werden als äußerer Sachzwang vorausgeseizt und nicht mehr als Resultat der sich wechselseitig beeinflussenden 
werden, um bei vorgegebenen und nicht beeinflussbaren Preisen (Warenpreise, Wechselkurse, Zinsen) am Ende einer »langen Welle der Konjunktur«, wenn Märkte stagnieren und ein inzwischen weltweiter Rationalisierungswettlauf ausbricht, mit den Konkurrenten mithalten zu können. Kein Wunder, daß der Lohn zur strategischen Eingriffsvariablen der Wirtschaftspolitik wird (dazu: Altvater/Mahnkopf 1993). Der Nationalstaat wandelt sich in dieser Lage in einen "Wettbewerbsstaat « (der Begriff ist von Hirsch 1994 glücklich gewählt), der »Standorte « gegen andere »Standorte « sichert. Das ist so lange kein Problem, wie auf dem expandierenden Weltmarkt alle in einem »Positivsummenspiel« gewinnen können. Wenn aber »Nullsummenspiele« oder gar »Negativsummenspiele« die Regeln bestimmen, wird Standortpolitik zu einer ruinösen und gefährlichen Strategie der Externalisierung von Kosten. Darauf wird zurickzukommen sein.

Die Nachkriegsordnung hat keine 50 Jahre gewährt. Der Fall der Mauer in Berlin 1989, spektakuläres Fanal des Kollapses des »real existierenden Sozialismus «, brachte das Ende der Bipolarität. Seitdem ist von einer »neuen Weltordnung « die Rede. Die Welt des ausgehenden 20. und beginnenden 21. Jahrhunderts gehorcht nach dem »Sieg im Kalten Krieg« und »am Ende der Geschichte « scheinbar alternativlos dem Prinzip der rationalen Weltbeherrschung durch Prozesse, die ökonomisch vom Markt und politisch von formal-demokratischen Verfahren gesteuert werden ( Vgl. die Diskussion über das »Posthistoire « bei Niethammer 1990 und Anderson 1992). Allerdings hat die Verfolgung des Prinzips der Weltbeherrschung viele Fragen aufgeworfen, auf die in der "neuen Weltordnung « neue Antworten gefunden werden müssen, nachdem sich die »alten« Antworten aus der Epoche von Bipolarität und Bretton Woods als unzureichend oder gar kontraproduktiv herausgestellt haben: Wie soll mit dem Scheitern der Entwicklungsanstrengungen der vergangenen Dekaden im politischen (d.h. nicht unbedingt geographischen) Süden des Globus umgegangen werden? Welche Regelwerke müssen vereinbart werden, um die außer Kontrolle geratenen internationalen Finanz- und Währungsbeziehungen zu »ordnen«? Können Modernisierung und Industrialisierung nach dem Muster des »nördlichen Westens« auch in Zukunft ein Ziel für alle Gesellschaften in allen Weltregionen des »post-sozialistischen« Ostens und des »unterentwickelten « Südens sein? Können alle Nationen die »Wettbewerbsfähigkeit« von $\gg$ Standorten« verbessern oder ist dies nur einigen, zumeist auf Kosten anderer möglich? Wie ist darauf zu reagieren, daß gleichzeitig mit der Perfektionierung der rationalen Weltbeherrschung die Natur »zurückschlägt«,

nationalstaatlichen Kräfte erfaßt. Tatsächlich wird noch vom »Nationalstaat« geredet, wo das Konzept der Nationalität des Wettbewerbsstaats gemeint ist. 
daß die globalen Ökosysteme - Wasser, Luft, Land und Eiskappen - aus dem Gleichgewicht zu geraten drohen und der »rationalen Weltbeherrschung « mit der » Tücke des Objekts« spotten? Die Zauberlehrlinge haben zwar den Besen in Bewegung zu setzen vermocht, können ihm aber nicht mehr Einhalt gebieten. Fatalerweise dürfen sie dies noch nicht einmal, wenn sie in der internationalen Konkurrenz stehen und bei einem Alleingang Nachteile zu gewärtigen haben. Unter dem Wettbewerb der Zauberlehrlinge leidet die Natur.

In der »neuen Weltordnung « nach der Bipolarität sind also alte Probleme immer noch ungelöst, und neue sind hinzugekommen. Zur besseren Orientierung können die Koordinaten der neuen Weltordnung mit Ortsnamen beschildert werden (Lipietz 1993): In Berlin fiel die Mauer; das war das telegene Bild vom Kollaps des Realsozialismus, der sich mit der samtenen Revolution in Prag, mit Glasnost und Perestroika in Moskau, an den runden Tischen in Warschau und mit der Protestbewegung in Budapest schon längst angekündigt hatte. Systemwettbewerb und Blockgegensatz waren plötzlich vorbei, und die bittere Phase der Transformation ehemals realsozialistischer Gesellschaften in Markt- und Geldwirtschaften und parlamentarische Demokratien konnte beginnen. Sofern der Begriff der »neuen Weltordnung « überhaupt Sinn macht, wird ihre Stabilität und Entwicklungsfähigkeit davon abhängen, ob Spielräume für Entwicklung geschaffen und Ressourcen bereitgestellt werden, um die langwierige und konfliktreiche Transformation von realsozialistischen Gesellschaften und deren Integration in den Weltmarkt zu einem positiven Abschluß bringen zu können. Also wird der Transformationsprozeß sich nicht auf die östlichen Länder beschränken. In einer hochgradig interdependenten Welt sind alle Gesellschaften, auch im Westen und Süden, in den TransformationsprozeB des Ostens einbezogen.

In der Dämmerung des heraufziehenden Nord-Süd-Gegensatzes ist das Weichbild Bagdads erkennbar, so wie auf den echtzeitigen CNN-Bildern der Raketenangriffe von 1991. Während des Aufmarsches der US-Truppen in der saudischen Wüste fiel im September 1990 der Begriff der »neuen Weltordnung « zum ersten Mal. Statt Entwicklung, die Präsident Truman in seiner »State of the Union« 1947 allen Ländern versprochen hatte, offeriert Präsident Bush in seiner $»$ State of the Union« 44 Jahre später im Februar 1991 nichts weniger als eine neue Weltordnung, ein neues »amerikanisches Jahrhundert $\ll^{4}$.

4 Paul Kennedy (1993, S. 370) weist darauf hin, daß der Begriff im Jahre 1941 von Henry Luce kreiert worden ist. Er fügt hinzu, daß dieser Ausdruck, gleichgültig ob er berechtigt und zutreffend sein mag, »eine immense psychologische und kulturelle Macht (besaß) und dem »amerikanischen Volk eine emotionale Unterfütterung seiner politischen Rolle« 
Der dritte Ort, Rio de Janeiro, steht für die beginnende Erkenntnis von der ökonomisch-ökologischen Paradoxie. Die westfälische Ordnung scheiterte am Streben von Nationalstaaten über die territorialen Grenzen hinaus. In der Nachkriegsordnung ist zwar die nationalstaatliche Grenzüberschreitung weitgehend gebannt. Doch andere Grenzen werden mißachtet: Auf der einen Seite eine Ordnung mit Wachstum ohne Grenzen, das die Attribute von Modernisierung, Industrialisierung und Konsumgesellschaft zur Verfügung stellt. Auf der anderen Seite das gesicherte Wissen um die Grenzen der Belastbarkeit der globalen Ökosysteme - und um die Notwendigkeit, daß neue politische Regelwerke für den Umgang der Menschen mit der Natur gefunden werden müssen. Die neuen Regeln und Prinzipien haben schon einen Namen: Er heißt »sustainability«.

Berlin symbolisiert das Ende der alten Ordnung der Regulation des OstWest-Gegensatzes. Bagdad bezeichnet den Umschwung zur neuen Ordnung der Kollusion ehemaliger Systemgegner und die Heraufkunft eines neuen Nord-Süd-Gegensatzes. Rio steht für mögliche Prinzipien des Neuen und Gemeinsamen in der »neuen Weltordnung «, da jenseits aller Konflikte eine Regelung des Zugriffs auf die globalen Ressourcen und Senken gefunden werden muß.

Die entscheidende Frage ist natürlich, ob das in Rio in Gang gesetzte Projekt der Regulation von Ressourcenverbrauch und Ressourcenbelastung unter der Zielsetzung »nachhaltigen« Wirtschaftens von den unter harten Zwängen der Weltmarktkonkurrenz stehenden »Wettbewerbsstaaten « überhaupt seriös verfolgt werden kann, ob nicht wie im Fall des Zauberlehrlings die Verfolgung des Programms rationaler Weltbeherrschung, zumal im Wettbewerb mit anderen Zauberlehrlingen, im allgemeinen Desaster endet.

\section{In Verfolgung des Programms »rationaler Weltbehereschang «}

Auch wenn der Ursprung des Prinzips der »rationalen Weltbeherrschung « weit zurück in der Geschichte liegt und insbesondere in den monotheistischen Religionen des östlichen Mittelmeers aufgespürt werden kann 5 , fand

(S. 371) gab. Nach dem »Sieg im Kalten Krieg« schien es so, als ob am »unipolar moment« einer unbefragten Hegemonie der USA angeknüpft werden könnte - wie in der alten Weltordnung nach dem 2. Weltkrieg.

5 Der Vers 1. Mose, 1, 28 lautet: »Und Gott segnete sie und sprach zu ihnen: Seid fruchtbar und mehret euch, und füllet die Erde, und macht sie euch untertan, und herrschet über Fische im Meer und über Vögel unter dem Himmel und über alles Tier, das auf Erden kreucht..." Und in Mose 1, 9,1 wird nach der Sintflut in der göttlichen Botschaft an Noah wiederholt: »Seid fruchtbar, und mehret euch, und erfüllet die Erde. « John Locke hat mit Bezug auf Psalm 115, 16 das Sondereigentum von Privaten an der allen Menschen gleicherweise zustehenden Schöpfung begründet: »Der Himmel allenthalben ist des Herrn, 
die »Weltbeherrschung « realiter über Jahrtausende nur lokal und regional (z. B. in den engen Grenzen der Polis, an den Gestaden der großen Binnenmeere) statt, und sie war im modernen europäischen Sinne keineswegs immer »rational «. Hannah Arendt zeigt, daß erst die Verwandlung des animal laborans in den homo faber die Herrschaft über die Welt begründet, und daß die antike Beziehung zwischen Mensch und Welt, in der die Unvergänglichkeit des Kosmos und die Vergänglichkeit des einzelnen im Zentrum stand, durch die christliche Heilslehre von der Unsterblichkeit des Einzellebens und der Vergänglichkeit der Welt abgelöst werden muß, um rechtfertigen zu können, daß und warum die Welt den Menschen untertan zu machen ist ${ }^{6}$.

Umweltschädigungen hatten wegen der eng gezogenen Grenzen des menschlichen Zugriffs auf die globalen Ressourcen nur lokale oder regionale Reichweite. Der Smog über Athen, die Abwasserplage und der Gestank der Abfälle in Rom, die Abholzung und nachfolgende Verkarstung der Balkangebirge, der Untergang der Kultur der Osterinseln waren Folgen tiefgreifender Einschnitte in regionale Ökosysteme, für die Funktionsweise des Ökosystems Erde jedoch waren sie eher belanglos. Zu einem globalen Prinzip wird die rationale Weltbeherrschung erst in der Neuzeit, als die »marinheiros « der iberischen Halbinsel bei dem schon vorher (z. B. von den Wikingern) unternommenen Versuch Erfolg hatten, die »Nahtlinien

aber die Erde hat er den Menschenkindern gegeben«. Im Koran heiBt es in der Sure AlBaqarah, Vers 23: »Der die Erde gemacht hat zu einem Bette für euch, und den Himmel zu einem Dach, und Wasser hat niederregnen lassen von den Wolken und damit Früchte für euren Unterhalt hervorgebracht hat. Stellt Allah daher keine Götter zur Seite, denn ihr wißt es doch. « Wer den Menschen so viel bietet, kann für sich beanspruchen: »Du sollst keine anderen Götter neben mir haben« (2. Mose, 20, 3). Der monotheistische Monopolanspruch und der Auftrag der expansionistischen Weltbeherrschung sind Kehrseiten eines einzigen zivilisatorischen Projekts, das sich als ungeheuer geschichtsmächtig herausgestellt hat.

6 Sehr schön schreibt Hannah Arendt in »Vita Activa«: »Die Bibel hat Adam, den dem Akker verpflichteten, arbeitenden Menschen, zum Herrn über alle lebende Kreatur gesetzt, aber das Animal laborans, das die Kraft des eigenen Körpers durch die Kraft der ihm unterstellten und von ihm gezähmten Tiere vervielfachen kann, um dem Leben seine Nahrung zuzuführen, wird nie Herr der Erde und der Natur selbst. Nur weil er auch Homo faber ist, kann es dem Menschen gelingen, Herr und Meister der gesamten Erde zu werden. Und da menschliche Produktivität sich immer an der göttlichen Schöpferkraft gemessen hat, die ex nihilo, aus dem Nichts schafft, während der Mensch eine Substanz braucht, die er gestaltet, hat sich das Bild der Rebellion des Prometheus der Vorstellung von Homo faber so innig vermählt... (Arendt 1981: 127). Zum Übergang vom antikeñ zum christlichen Weltbild schreibt sie: »Denn die christliche Heilsbotschaft von der Unsterblichkeit des Einzellebens verkehrte ihrem Wesen nach die antike Beziehung zwischen Mensch und Welt; da, wo antikem Glauben zufolge die Unvergänglichkeit des Kosmos gestanden hatte, erschien nun ein unsterbliches menschliches Leben, und an den Platz, den die Sterblichen eingenommen hatten, rückte nun eine vergängliche Welt.« (Arendt 1981, S. 306) 
der Pangäa« (Crosby 1991), die ozeanischen Wasserscheiden zwischen den kontinentalen Stücken des einstmals einheitlichen Urkontinents Pangäa, von Europa aus zu überschreiten. Die »neo-europäischen« Kolonien jenseits der Ozeane wurden okkupiert und kolonisiert und der europäischen Umgangsform mit der innerern und äußeren Natur unterworfen. Im übrigen wanderten nicht nur Menschen von Europa in die fernen Kolonien. Sie haben ihre Tiere, Pflanzen und Krankheiten mitgenommen, die sich zum Teil ebenso unaufhaltsam und opportunistisch des Landes bemächtigten und andere Lebewesen als Konkurrenten verdrängten wie die »Herren der Schöpfung « es getan haben (dazu ausführlich Crosby 1991). Im Verlauf der gewaltsamen Europäisierung der Welt ist die ursprüngliche Mannigfaltigkeit der Arten beirächtlich reduziert worden. Folglich ist die Bahn der Evolution seit den großen Entdeckungen europäisiert worden.

Anders als in den Jahrhunderten zuvor sind heute alle Weltregionen in den Bann des ökonomischen Prinzips von Expansion im Raum und Wachstum in der Zeit geschlagen. Weiße Flecken auf der Weltkarte gibt es nicht mehr, der Kreis »zivilisierter« Nationen, der »charmed circle« definiert sich nicht mehr in Abgrenzung zur »unzivilisierten Welt«, zu der von Sir Thomas Holland noch in den 20er Jahren Länder wie China oder Persien gezählt wurden (Rigaux 1991, S. 388f). Damit das Prinzip der rationalen Weltbeherrschung aus der Welt des Geistes, aus Religion und Philosophie in die materiale Welt eintreten und zur Maxime des praktischen Handelns werden konnte, mußten sich die materialen und sozialen Verhältnisse ändern. Dies passiert zu Beginn der Neuzeit. Am Ausgang der Krise des europäischen Mittelalters, die von der zweiten Hälfte des 14. bis zur zweiten Hälfte des 15. Jahrhunderts dauerte und die europäische Bevölkerung mehr als dezimierte (dazu Sieferle 1989), bildet sich, gestützt auf neue Produktivkräfte und neue Energiesysteme (z. B. Wasser- und Windmühle), eine neue soziale Formation (Debeir u.a. 1989, S. 118ff). Sie birgt bereits den Keim der später entstehenden modernen kapitalistischen Produktionsweise in sich. Der Übergang von der Natural- zur Geldrente, noch unter feudalen Verhältnissen, leitet die von Marx so bezeichnete $\gg$ ursprüngliche Akkumulation des Kapitals« (MEW 23, S. 741ff) ein, jenen sozialen Scheidungsprozeß, an dessen Ende sich Kapital und Arbeit und - nach der Verselbständigung des zinstragenden Kapitals - Profit, Lohn und Zins gegenüberstehen.

Die Knospen der Produktionsweise brechen mit der industriellen Revolution zur Blüte auf. Als die fossilen Energieträger (zunächst Kohle, später Erdöl) nicht mehr nur zum Kochen und Wärmen, sondern zur Erzeugung von Energie zur Arbeitsleistung systematisch genutzt werden, können die natürlich begrenzten biotischen Energien - wiederum ganz natürlich - 
enorm gesteigert werden?. Energiewandlungssysteme (dazu gehört die Dampfmaschine), die erstens die in der Kohle (später im Erdöl) gespeicherte Energie in nützliche Endenergie (z. B. Bewegungsenergie) verwandeln können und zweitens wesentlich mehr Nutzenergie ernten (Kohlen aus der Erdkruste brechen und fördern) als sie als Input einsetzen müssen, räumen die unüberwindlich erscheinenden Hindernisse einer Steigerung der Produktivkräfte aus dem Weg. Die biotischen, sendosomatischen « Kräfte von Mensch und Tier sind natürlich begrenzt. Die Geschwindigkeit eines Ochsen, eines Pferdes, eines Menschen ist trotz des Strebens nach Weltrekorden über das tierische oder menschliche Maß hinaus nicht steigerungsfähig. Der Rückgriff auf fossile Energieträger, auf »exosomatische Kräfte« (Lotka) bedeutete daher einen Quantensprung bei der Steigerung der Geschwindigkeit, bei dem Zugewinn an räumlicher Mobilität und Reichweite, bei der Erhöhung der Arbeitsproduktivität, der Produktenmenge und damit des »Wohlstands der Nationen«. Nicholas GeorgescuRoegen (1986) spricht in diesem Zusammenhang von einer "prometheischen Revolution « in den Methoden der Produktivitätssteigerung, die die (zunächst europäische, später die neo-europäische) Menschheit mit der Umstellung ihres Energiesystems, der Produktionsweise, der sozialen Organisation durchgemacht hat. Marx zeigt in seiner Analyse des »Produktionsprozesses des Kapitals«, wie das Ensemble von Bewegungs-, Transmissions- und Werkzeugmaschine in der »großen Industrie « die nicht mehr nur »formelle «, sondern die »reelle Subsumtion der Arbeit unter das Kapital« zur Produktion des relativen Mehrwerts ermöglicht (MEW 23, S. 331ff). Das Grundprinzip heißt Steigerung der Produktivität der Arbeit; dessen Realisierung setzt ebenso die Verfügung über fossile Energieträger voraus, wie die Fähigkeit, damit zur Steigerung der Produktion je Arbeiter sinnvoll umgehen zu können. Also verwandelt sich in diesem Prozeß die Gesellschaft. Die energetische ist eine soziale Revolution.

Nun erst kann Weltbeherrschung richtig ausgelebt werden. »Macht Euch die Erde untertan! « war bislang göttliches Gebot, das nur menschlich, und das heißt unvollkommen befolgt werden konnte. Nun aber wurde es zur realisierten, weil realisierbaren Maxime. Europa expandiert über seine vertrauten Grenzen hinaus, und die Aktivitäten der Menschen in Produktion und Reproduktion beschleunigen sich. Die Regel, daß die Zeitporen die

7 Prigogine und Stengers beschreiben die »amüsante Tatsache, daß Adam Smith an seinem Reichtum der Nationen arbeitete..., als James Watt an der gleichen Universität dabei war, letzte Hand an seine Dampfmaschine zu legen. Dennoch sieht Adam Smith in seinem Buch den einzigen Nutzen der Kohle darin, Heizwärme für die Arbeiter zu liefern« (Prigogine/Stengers 1986, S. 111). Auch an den schottischen Universitäten des späten 18. Jahrhunderts war es offenbar nicht leicht, Interdisziplinarität zum praktischen Prinzip zu erheben. 
Elemente des Gewinns darstellen und daher »time money« ist, kann zu einem dominanten Prinzip werden, das den sozialen Wandel selbst in nichtrevolutionären Zeiten beschleunigt. Das Kapital strebt »eine Form der Cirkulation ohne Cirkulationszeit « (Marx, MEGA, II 1.2, S. 543) an, also die zeitlose und auch raumlose Ökonomie und Gesellschaft, die in den neoklassischen Modellen so selbstverständlich und daher so unkritisch vorausgesetzt wird. Nichts bleibt stationär. Eine statische oder komparativ-statische Theorie ist ungeeignet, das Neue zu begreifen: Der Wandel wird zur Normalität; Kontinuität hingegen ist Stagnation, ist Krise.

"Eine grundlegende Kontinuität charakterisierte die vorindustrielle Welt, selbst nach so großen Umwälzungen wie dem Aufstieg und Fall des römischen Reiches, des Islam und der chinesischen Dynastien... Diese Kontinuität wurde zwischen 1750 und 1850 unterbrochen « (Cipolla 1985, S. 2$)^{8}$.

Nun wird der Wandel zum Prinzip, das jedem einzelnen Menschen permanente Anpassungsleistungen abfordert. Das Fatale, Verrückte und Tragische der Weltbeherrschung besteht ja darin, daß niemand in diesem Projekt zur Ruhe kommen kann. Rastlosigkeit, Verlust der Heimat (im Sinne Blochs) und von sozialen Bindungen sind die Kehrseiten des Triumphs der Weltberherrschung. In der gegenwärtigen Debatte über Flexibilität, Mobilität und Deregulierung wird der permanente Wandel und die flexible, mobile, interesselose Anpassungsfähigkeit zur Tugend verklärt und zur Verinnerlichung empfohlen. Gesellschaften werden aber ihrer sozialen Bindungskraft beraubt, wenn die Trägheitseigenschaften von räumlicher Verortung und von routinierten Zeitregimen unterdrückt werden ${ }^{9}$. Es lösen

8 Cipolla unterstreicht die revolutionäre Veränderung im Zuge der Industrialisierung mit einer Bemerkung C.H. Waddingtons aus dem Jahre 1960: "Wenu ein alter Römer achtzehn Jahrhunderte später wieder auf die Welt gekommen wäre, hätte er sich in einer Gesellschaft wiedergefunden, die er ohne Schwierigkeit verstanden hätte. Horaz wäre sich als Gast bei Horace Walpole nicht deplaciert vorgekommen, und Catull hätte sich zwischen den Wagen, Damen und brennenden Lampen nachts im London des 18. Jahrhunderts zu Hause gefühlt. « Er fügt dem Zitat hinzu: »Wenn ein General in der Mitte des 19. Jahrhunderts die Organisation des römischen Heeres studierte, wenn ein Arzt sich mit Hippokrates oder Galen beschäftigte, wenn ein Agronom Columella las, so tat er das aus rein geschichtlichem Interesse und akademischer Neugier. Selbst im fernen, unwandelbaren China wurde es den Aufgeklärtesten der literarisch gebildeten Bürokraten klar, daß die klassischen alten Autoritäten, die der chinesischen Geschichte über alle Invasionen und Dynastiewechsel hinweg Kontinuität verliehen hatten, für das Überleben in der modernen Welt nicht länger Gültigkeit hatten. 1850 war die Vergangenheit nicht nur vergangen - sie war tot « (Cipolla 1985, S. 2).

9 Sindbad der Seefahrer erlebt in einem für heutige Verhältnisse kleinen regionalen Raum, an den Gestaden des persischen Golfes, an den Küsten des indischen Ozeans, die überraschendsten Abenteuer und mußte den Umgang nicht nur mit ganz unterschiedlichen Kulturen und Techniken, sondern auch mit unbekannten, gefährlichen Lebewesen (dem Nashorn, dem Vogel Ruch) lernen, um seinem im übrigen einträglichen Geschäft des Fernhandels nachzugehen. Ein schönes Märchen nach dem Krieg, nach brennenden Ölfeldern und verseuchten Stränden des Jahres 1991. Die dramatischen Geschehnisse der Odyssee 
sich freischwebende Sozialisationspotentiale, die dem populistischen und autoritären Zugriff vergleichsweise einfach zur Verfügung stehen.

Die Welt ist ein einheitlicher ökonomischer und ökologischer Raum geworden, ein globaler Basar, wo überall dort, wo sich monetäre Kaufkraft konzentriert, jene Zitronen zu haben sind, von denen Goethe träumte. Dies macht den Bruch mit der langen »Vorgeschichte aus, die von der Profanität industrieller Verhältnisse abgelöst wird. Nichts ist wie früher. Am Ende der »great transformation « (Polanyi) zur Marktwirtschaft in Verbindung mit der »prometheischen Revolution« der Industriegesellschaft (Georgescu-Roegen), unterstützt von den »bürgerlichen Revolutionen « in England, Frankreich und in den USA, durch die den Menschenrechten und dem demokratischem Prinzip eine Bresche geschlagen wurde (Hannah Arendt), ist die moderne kapitalistische Produktionsweise mit ihrer »propagandistischen Tendenz, den Weltmarkt herzustellen « (Marx), nicht nur hervorgebracht, sondern siegreiches, und - wie viele meinen - alternativloses Prinzip der gesellschaftlichen Organisation des Stoffwechsels zwischen Mensch und Natur.

Von Schumpeter stammt das Wort, daß ein industrielles Unternehmen nicht für den Bedarf von Königinnen, sondern für den von Arbeiterinnen produziert. Er hat Recht. Industrie bedeutet Massenproduktion und daher auch Massenkonsum. Den sozialen Mechanismus und die ökonomischen Methoden entwickelt zu haben, um Massenproduktion und Massenkonsum etwa im Gleichschritt zu steigern, ist die große historische Errungenschaft des Fordismus als sozialer Form (Aglietta 1979; Hurtienne 1986; Hübner 1989) und von Keynesianismus als wirtschafts- und sozialpolitischem Projekt. Das industrielle System und erst recht seine fordistische Form ermöglicht daher - im Prinzip! - nicht nur mehr Wohlstand, sondern auch mehr soziale Gleichheit als jedes andere Gesellschaftssytem zuvor. Dies macht ja einen Gutteil der Attraktivität des industriellen Kapitalismus aus.

finden zwischen der Ostküste Griechenlands und der Westküste Italiens, zwischen ägäischem und thyrennischem Meer in einem Raum statt, der heute für Kreuzfahrten gestreßter Kurzurlauber gut ist. Als Goethe Ende der 80er Jahre des 18. Jahrhunderts nach Italien reiste, brauchte er nicht nur sehr viel Zeit. 57 Tage dauerte die Fahrt von Karlsbad nach Rom. Sicher, er hätte sie verkürzen können. Aber 12 Tage wären in jedem Fall das Minimum bei größter Hetze gewesen. Das »Land, wo die Zitronen blühen«, war fern, einmalig und daher wunderbar zugleich. Der Reiz ist bei einer Flugreise von knapp zwei Stunden zwischen Frankfurt und Rom dahin. Goethe mußte noch sehr viele Zoll- und Währungsgrenzen passieren und die Reise durch Bankanweisungen oder gut versteckte Silber- und Goldmünzen - denn die Gefahr ausgeraubt zu werden, war nicht klein - vorbereiten (Klauss 1989). 200 Jahre später wird Europa zu einer Währungsunion zusammengeschmiedet, Zollgrenzen zwischen Italien und den deutschen Landen gibt es nicht mehr. »Als Wieland 1772 von Erfurt nach Weimar kam, schien ihm dieser Ortswechsel 'einer Emigration in eine neue Welt ähnlich'« (Klauss 1990, S. 15). Heute werben die beiden 24 km entfernten Städte um Kunden für ihren gemeinsamen Nahverkehrsverbund... 
Soziale Gleichheit und demokratische Teilhabe jedoch, so Ernest Gellner (1991), haben eine entropische Komponente: sie lösen traditionelle soziale Differenzen, Status- und Gruppenidentitäten oder Privilegien auf 10 . Die soziale Entropie steigt mit der größeren sozialen Gleichheit - und mit ihr die Versuche, der $\gg$ Nivellierung « in der »Mittelstandsgesellschaft« zu entgehen.

Für diese Versuche sind zwei systembedingte Gründe verantwortlich. Zum einen erzwingt das Prinzip der Konkurrenz nicht nur entropischen Ausgleich, sondern auch Ungleichheit. Seine Wirkungsweise, darin besteht der Sinn des Wettbewerbs, verwandelt gleiche Ausgangsbedingungen in ungleiche Resultate, die in der »nächsten Runde« ungleiche Ausgangsbedingungen darstellen. Der andere Grund hat mit der Individualisierung zu tun, die als Tendenz der Moderne soziale Gleichheit ebenso voraussetzt wie immer wieder auflöst. Im Produktionsprozeß sind in jedem Fall gemeinsame Klassenlagen relevant, für die Arbeiter als Produzenten sind »Milieus « bis in die heutige Zeit bestimmend. Als Konsumenten jedoch sind sie mit Geld ausgestattete »souveräne « Individuen, die ihre eigenen Lebensentwürfe $\mathrm{zu}$ gestalten versuchen. Neue Ungleichheiten entstehen und reproduzieren sich also durch Konkurrenz und Individualisierung.

\section{Der Bund von Kohle und »Kohlen«}

Die »Rationalität der Weltbeherrschung « findet nicht nur Produktivkräfte vor, die der bloßen Idee oder dem göttlichen Gebot zur geschichtsmächtigen Realisierung verhelfen. Vielmehr »drängt die Wirklichkeit « mit der Umwälzung von sozialen Formen »zum Gedanken«, sie helfen der »prometheischen Revolution« historisch auf die Sprünge. Geld ist dabei die entscheidende soziale Form und das Medium der Ökonomie. Der "Quantitativismus des Geldes«, den Aristoteles noch kritisierte, weil er sozial zersetzend wirke, kann sich so recht entfalten, wenn die technischen Wandlungssysteme von Energie und Stoff die räumlichen und zeitlichen Grenzen des »Oikos« und der »Polis« weit hinter sich lassen und die (endosomatischen) Kräfte von Mensch und Tier (exosomatisch) vervielfachen. Für die moderne Geldwirtschaft, die bereits im 14. Jahrhundert in

10 Hierbei handelt es sich um ein Thema, das von Antoine-Augustin Cournot aufgeworfen wurde: Einerseits führt Rationalisierung zu einer Art endgültigem Zustand der Zivilisation, andererseits die Herausbildung des Marktgleichgewichts zu einer Vereinheitlichung »atomisierter « und »pulverisierter« Agenten, deren soziale und individuelle Differenzen unter der Betonung der Einzigartigkeit jedes Atoms oder Pulverkörnchens in »kristallinen Formen« verschwinden (vgl. Anderson 1992: 294ff). Arnold Gehlen hat Cournots Gedanken aufgegriffen und zur These von der »industriegesellschaftlichen Erosion « fortgeführt, ihm dabei aber den kritischen Impuls genommen, auf den Anderson (ebenda) verweist. 
den oberitalienischen Stadtrepubliken entsteht, sind die fossilen Energieträger der prometheischen Revolution ein Treibsatz, der sie in den siebten Himmel heutiger globaler Finanzspekulation emporjagt. Mit den Transport- und Kommunikationsmedien des 20. Jahrhunderts wird der zeitlichen und räumlichen Dynamik des Geldes und des Kapitals Tür und Tor geöffnet. Die hinderlichen Transport- und Transaktionskosten werden auf ein Minimum reduziert, wenn »Kohlen« und Kohle einen Bund fürs Leben, zumindest der Industriegesellschaft, bilden. Ohne fossile Energien gäbe es weder den kapitalistischen Produktions- und Akkumulationsprozeß noch den modernen monetären Weltmarkt. Wie sonst könnten in Sekundenbruchteilen Milliarden Dollar von Hong Kong nach New York oder von Tokio nach London transferiert werden? Erst weil Raum und Zeit durch den »technischen Fortschritt « geradezu vernichtet worden sind, so daß von der Ökonomie gedankenlos als einer »virtuellen « Veranstaltung gesprochen wird (Menzel 1993), müssen »Standorte « zwischen La Plata, Rio Grande, Rhein, Po und Wolga, mit höchst reellen Leistungen - Lohnkosten, Infrastruktur, Kompetenz staatlicher Verwaltungen etc. - um investierbare liquide Fonds auf dem »virtuellen « monetären Weltmarkt buhlen.

Ohne die Möglichkeiten, die Produktivität der Arbeit mit Hilfe fossiler Energieträger, technischer Wandlungssysteme in einer angemessenen sozialen Form zu steigern, ohne wirtschaftliches Wachstum also, würde die Erhebung eines Zinses auf ausgeliehenes Geld die ökonomische Substanz von Schuldnern sehr schnell auszehren. Zinsen könnte es gar nicht auf Dauer geben, wenn es nicht gelungen wäre, einen ökonomischen Überschuß zu produzieren und diesen quantitativ zu steigern, indem die qualitativen Produktionsbedingungen bei der »relativen Mehrwertproduktion« umgewälzt werden. Der Überschuß nimmt in kapitalistischen Verhältnissen die Wertform an (Mehrwert), ist aber von der materiellen Seite nicht losgelöst. Denn der »Träger des Werts«, so Marx, »ist der Gebrauchswert«. Der Zusammenhang ist höchst vermittelt, aber er existiert. Diese letztliche Bindung monetärer Größen an reale (und soziale) Verhältnisse ist der rationale Kern der Warnung von Aristoteles, von Augustinus und Thomas von Aquin vor der sozial zersetzenden Wirkung des Zinses, daher das bis heute formal gültige Zinsverbot im Islam. Sind die Grenzen der biotischen Energien aber durch die Einführung fossiler Energieträger aufgehoben, ist ein nicht mehr an Tempo und Grenzen organischer Wachstumsprozesse gebundener Zuwachs der Produktion ebenso möglich wie die Erzielung von (monetären) Überschüssen. Zinsen können erhoben und gezahlt werden, ohne daß dadurch das Gemeinwesen sozial zersetzt oder Individuen in den Bankrott getrieben werden. Im Gegenteil, der Zins übt als »monetäre Budgetrestriktion« eine stimulierende Wirkung auf die Produktion aus: Er 
zwingt den Kreditnehmer zur Steigerung der Arbeitsproduktivität, also zu Innovation in Technik, Arbeitsorganisation und Managementstrategien. Fossile Energieträger und Geld gehen in der sozialen Formation des Kapitalismus eine synergetische Beziehung ein, sie steigern wechselseitig ihre jeweilige Dynamik. Ohne Geld schlummerten Kohle und Öl noch in der Erdkruste, und ohne die Kraft der fossilen Brennstoffe wäre der »Quantitativismus des Geldes « eine harmlose Geschichte ${ }^{11}$.

Zeitversetzt bildet sich nach der "prometheischen «, industriellen und energiewirtschaftlichen Revolution des ausgehenden 18. Jahrhunderts eine globale Rohstoff- und Energiewirtschaft heraus. Während in vormodernen Zeiten die Menschen nur in räumlich begrenztem Maße zum Transport der Energieträger (in erster Linie des Holzes) in der Lage waren (Debeir u.a. 1991) und daher nach dessen Verfügbarkeit ihre »Standorte « ausrichten mußten, verschaffen sich moderne kapitalistische Gesellschaften Energien und Rohstoffe aus allen Weltregionen, um sie in den Zentren der Energieund Stoffwandlungssysteme in jenes Ensemble von Gebrauchswerten zu verwandeln, das die Schaufenster der »modernen Wohlstandsgesellschaft « füllt und den »Reichtum der Nationen« darstellt. Im »global sourcing« von Energiemultis ist dieser Tendenz die entsprechende Strategie unterlegt worden. Dies alles setzt Speicherbarkeit über längere Zeiträume, leichte Transportierbarkeit und problemlose Transformierbarkeit von Primär- in Sekundär- und Endenergien (aus Kohle in Wärme, in Bewegung und umgekehrt aus Bewegung in Wärme und in Elektrizität) voraus. Diese Bedingungen werden von den fossilen Energieträgern hervorragend erfüllt. Wegen des möglichen Zugriffs auf Lagerstätten in aller Welt können Industriestandorte nach ganz anderen Kriterien als der Verfügbarkeit von Rohstoffen ausgewählt werden. »Just-in-time« oder »lean production« sind erst Optionen geworden, als die Rohstoffwirtschaft internationalisiert und der Gegensatz von Produktions- und Extraktionsorten entstanden ist. Der »Wettbewerbsstaat könnte seine Rolle auch mit noch so vielen »Kohlen« gar nicht spielen, wenn die Kohle ausbliebe. So ist der menschheitsgeschichtlich ungeheure Aufschwung der modernen Industriesysteme möglich geworden, indem die Gesellschaften in der Lage waren, Technik und soziale Institutionen zu »systematisieren« und die Anforderungen des Risikomanagements beim Umgang mit den exosomatischen Energien zu be-

11 Der Zusammenhang von Geld, Zins und fossilen Energieträgern, von monetären und energetischen Kategorien, ist für das Verständnis der Entwicklungstendenzen der kapitalistischen Industriegesellschaft äußerst wichtig. In der monetär-keynesianischen Theorie wird er vollständig ausgeblendet. Kein Wunder, daß Versuche, innerhalb der monetärkeynesianischen Theorie ökologische Fragen zu diskutieren, eher unbeholfen ausfallen und Anleihen bei den kritisierten Neoklassikern gemacht werden müssen (vgl. Betz u. a. 1993; S. $115 \mathrm{ff})$. 
wältigen (dazu: Altvater 1992, S. 47ff). Ein Risiko kann ja erst entstehen, wenn energetische Potentiale zu zweckgerichteter Arbeit konzentriert werden, die menschliche Maßverhältnisse extrem übersteigen und daher selbst nur mit komplexen technischen Apparaten und umfassenden gesellschaftlichen Vorkehrungen unter permanenter Kontrolle gehalten werden können. Doch Globalisierung bedeutet keineswegs, daß die Entwicklung der modernen Industriesysteme gleichzeitig und gleichmäßig erfolgen oder daß integrale Extraktions- und Produktionssysteme entstehen würden. Die »Weltordnung « der zweiten Hälfte des 20. Jahrhunderts ist vielmehr durch den Gegensatz von hoch kompetitiven Stoff- und Energiewandlungssystemen in den entwickelten Industriegesellschaften »des Nordens « einerseits und weniger entwickelten Rohstoff-Extraktionsländern »des Südens《 (»Syntropieinseln« nennt Hans Peter Dürr die Lagerstätten konzentrierter Rohstoffe) andererseits charakterisiert. Es ist keineswegs gesichert, daß gerade jene Länder und Regionen besondere Chancen haben, ökonomischen Wohlstand anzuhäufen, die über reiche Rohstofflager verfügen. Während im 19. Jahrhundert Rohstoffreichtum noch eine Gunsî des Standorts war, sind in der internationalen Arbeitsteilung des 20. Jahrhunderts eher jene Länder bevorzugt, die nicht auf Rohstoffexporte angewiesen sind, sondern technisch und organisatorisch innovativ mit neuen Produkten, produziert von qualifizierten Arbeitskräften, auf die Weltmärkte drängen können. Darauf basiert schon seit Jahrzehnten die Wettbewerbsfähigkeit der europäischen Industrieländer, dafür sind die »dynamischen Ökonomien« Asiens (nach Japan heute auch Suidkorea, Taiwan) aktuelle Beispiele. Rohstoffreiche und großflächige Länder wie Brasilien und Argentinien - in Zukunft vielleicht auch Rußland, Kasachstan oder die Ukraine - hingegen können mit den auf ihrem Territorium gelegenen $»$ Syntropieinseln « eher die industriellen Wandlungssysteme anderer Weltregionen beliefern und deren Energiehunger stillen, ais eigene - nicht mehr oder nur noch rudimentär vorhandene - Industrien versorgen. Mit den exportierten Energieträgern bieten sie den Industrieländern die Möglichkeit, ihren Wohlstand zu steigern, während nach der erfolgten Extraktion wenig mehr als ein »schwarzes Loch « (Euclides da Cunha über die ausgeplünderten Erzminen von Minas Gerais) uibrigbleibt.

\section{Extraktion versus Prodelation}

Der Ordnung der industriellen Welt entspricht die Unordnung der Extraktionsgebiete (dazu Altvater 1992 und 1993), es sei denn, Rohstoffländer schaffen es, die »penetrative power of the price system« (Innis 1956, S. 252ff) so zur Bildung interner Faktorpreisrelationen zu nutzen, daß Arbeit 
und Kapital aus dem Rohstoff- in den Industriesektor gelenkt werden. Allerdings müßten die Industrieprodukte konkurrenzfähig produziert und auf dem Weltmarkt angeboten werden können. Nur dann machte die Umlenkung von Faktoren aus dem Extraktions- in den Produktionssektor ökonomisch Sinn. Regionen und Nationen, die von der Extraktion und Vermarktung von »staple products« abhängen, sind jedoch benachteiligt. Sie müssen durch geeignete Wirtschaftspolitik das doppelte Problem lösen, bei gegebenen Faktorpreisrelationen auf dem Weltmarkt (terms of trade) die internen terms of trade so zu gestalten, daß »systemische« Wettbewerbsfähigkeit der verarbeitenden Industrien gegenüber dem Rohstoffsektor intern und gegenüber konkurrierenden verarbeitenden Industrien auf dem Weltmarkt extern hergestellt wird. Die »systemische Wettbewerbsfähigkeit « bezieht sich also immer auf die Konkurrenz innerhalb und zwischen den Branchen. Dieser Sachverhalt wird sehr häufig in den Analysen zur Wettbewerbsfähigkeit vernachlässigt ${ }^{12}$. Da steht immer die Konkurrenz innerhalb der Branche mit vergleichbaren Produkten aus anderen Ländern, produziert an anderen »Standorten $\ll$, im Vordergrund: Japanische gegen deutsche, US-amerikanische, italienische Autos; brasilianisches gegen australisches Erz usw. Die Gründe für die Schwierigkeiten, im jeweiligen Land den Faktortransfer »zwischen den Branchen« vom Rohstoffsektor in den Industriesektor zu bewerkstelligen, sind vielfältig und können hier nicht in gebotener Ausführlichkeit diskutiert werden ${ }^{13}$. Es wäre notwendig, die »alte« entwicklungstheoretische und -politische Debatte über endogene und exogene Blockaden von Industrialisierung und Modernisierung mit den »neuen« Ansätzen einer Theorie der Wettbewerbsfähigkeit, allerdings unter härter werdenden ökologischen Restriktionen, zu verknüpfen.

12 In der Marxschen Theorie »sorgt《 dic Konkurrenz innerhalb der Branche für den Ausgleich der individuellen Preise zu einem »Marktwert « und die Konkurrenz zwischen den Branchen für den Ausgleich der Profitraten. Die Konkurrenz mit Preisen (und Kosten) ist Gegenstand der Analyse der »Wettbewerbsfähigkeit« von Nationen, die profitgesteuerten Kapitalbewegungen zwischen den Branchen sind es in der Regel nicht.

13 Sie sind von Harold Innis (1930) in seiner Analyse der »staple products « (Kabeljau, Felle, Holz) am Beispiel Kanadas dargelegt worden (Vgl. auch Watkins 1981). Sie sind mit thermodynamischen Kategorien von Steven Bunker (1985) debattiert worden, um die Entwicklungshemmnisse in Amazonien (»underdeveloping the Amazon «) zu erklären (vgl. auch Altvater 1987; 1991). Hirschman hat auf die Notwendigkeit der Entstehung von »linkages« zwischen Branchen hingewiesen und auch darauf, daß die linkages nur unter besonderen Bedingungen zustandekommen (Hirschman 1981). Sie sind unter dem Etikett der »holländischen Krankheit « verkehrter Faktorpreisproportionen und klassenspezifischer Interessenlagen insbesondere am Beispiel von Ölländern diskutiert worden (dazu: Gregory 1976). Massarrat hat die Probleme der Entwicklungsblockaden mit »Manipulationen « des »Nordens« im »Dual-System der Weltökonomie« erklärt, mit denen verhindert worden sei, »daß Ressourcenbestände in den Entwicklungsländern des Suidens Kapitalcharakter annehmen« (Massarrat 1993: 46). 
Daß die Entwickungsunterschiede zwischen Ressourcen extrahierenden Rohstoffländern und den Ressourcen verwendenden Industrieländern überhaupt zum Problem geworden sind, liegt an der umfassenden, inzwischen durch die Kommunikationsmedien realisierten Globalisierung von Standards und Leitbildern von Produktion und Konsumtion. Entwicklung ist nicht mehr »nur« nationales Ziel der Förderung der produktiven Kräfte einer $\gg$ Nationalökonomie «, die Friedrich List vor 150 Jahren in Abgrenzung zu den hochgesteckten Erwartungen in die »komparativen Kostenvorteile « des Freihandels im klassischen »Kosmopolitismus « von Smith und Ricardo vorschwebte, sondern ein Versprechen im Rahmen der globalen Ordnung, Leitbild eines globalen Modells von Produktion und Konsumtion. Unterentwicklung ist daher nicht nur ein Problem, weil das Pro-Kopf-Einkommen niedrig ist und gemessen an anderen Gesellschaften angehoben werden sollte, sondern weil das Kriterium des Erfolgs, weil Modernisierung und Industrialisierung in vielen Weltregionen so eklatant verfehlt worden sind. Maßstab, Standards, Normen und Werte sind obendrein veränderlich. Die Latte wird, auch dies ist Ausdruck des Modells, von den jeweils Erfolgreichsten im Wettbewerb immer höher gehängt. Der Quantitativismus des Geldes bestimmt auch die Regeln und die Dynamik der internationalen Konkurrenz. Nichts bleibt wie es ist, alle Konkurrenten stehen unter dem Zwang, nicht nur die Latte zu nehmen, sondern sogleich die Maßstäbe höher zu schrauben. Das Prinzip Wettbewerb durchdringt auch staatliche Politik, der Staat wird zum »Wettbewerbsstaat « (Hirsch 1994) und organisiert die »Konkurrenzfähigkeit« von »Standorten«, unter denen Extraktionsorte und -regionen in aller Regel schlechte Karten haben.

\section{Wetthewerb und die Belastbarkeit der globalen Ökosysteme}

Hat dies etwas mit der globalen Ökologie zu tun? Konkurrenzfähigkeit und Entwicklungserfolge schlagen als vermehrte Produktion (unter besonderen Umständen eines »goldenen Zeitalters« der Akkumulation auch als zusätzliche Beschäftigung) zu Buche. Die Steigerung des Sozialprodukts in monetären Größen ist nur möglich, wenn auch der Verbrauch von stofflichen und energetischen Inputs steigt - selbst bei verbesserter Energieeffizienz ${ }^{14}$. So schürzt sich das - neben den begrenzten Ressourcen - andere Problem der globalen Ordnung: Durch die Nebenprodukte der Stoff- und Energie-

14 In der BRD (West) ist zwischen 1973 und 1993 die Wirtschaftsleistung um 50\%, die Kohlendioxid-Emission um 7\% gestiegen (DIW-Wochenbericht 9/94). Für andere OECDLänder gibt es ähnliche Werte (vgl. OECD 1991). Allgemein ergeben sich die Umweltauswirkungen von ökonomischen Aktivitäten aus der Bevölkerungsentwicklung multipliziert mit dem Pro-Kopf-Verbrauch von Gütern und Diensten multipliziert mit dem Technologieeffekt (Wissenschaftlicher Beirat 1993: 115). 
wandlung in der Produktions- und Konsumtionssphäre werden die natürlichen Senken für flüssige, gasförmige und feste Schadstoffe auf der Erde beansprucht - und schließlich uiberbeansprucht. Die Senken sind ebenso erschöpflich wie die Ressourcen, bei deren Umwandlung in gewünschte Gebrauchswerte sie entstehen. Die Frage ist also »ernst zu nehmen, ob nicht doch dem Energie- und Senkenproblem wachstumsbegrenzende Wirkung zukommt« (Wissenschaftlicher Beirat 1993: 158). Das ökologische Problem der globalen Ordnung kann also in ein »Ressourcen«- und in ein »Senken«-Problem aufgegliedert werden. Das erstgenannte Problem stand am Beginn der modernen Ökologie-Debatte, es wurde zum entscheidenden Thema des ersten Berichts des Club of Rome. Das zweite Thema kam später hinzu, ist aber möglicherweise wichtiger als das erste. Dabei muß allerdings berücksichtigt werden, daß die Unterscheidung im konkreten Fall nicht immer stichhaltig ist. Wälder beispielsweise sind »Ressourcen«. Holz (von anderen Waldprodukten abgesehen) kann als Rohstoff und Energieträger verwendet werden. Zugleich sind Wälder Senken für $\mathrm{CO}_{2}$ Emissionen, die durch die Biomasse im Wachstumsprozeß gebunden werden. Darüber hinaus sind sie - insbesondere die tropischen Regenwälder Lebensräume für eine Vielfalt und Vielzahl von Lebenwesen.

Die globalen ökologischen Probleme sind daher im Prinzip dreifacher Natur: (1) Erschöpfliche, endliche Ressourcen werden bis zur Neige ausgebeutet und prinzipiell nicht-erschöpfliche und erneuerbare Ressourcen werden über die Regenerationsfähigkeit hinaus (aus)genutzt. (2) Senken werden in einem Ausmaß belastet, das die Aufnahme- und Regenerationsfähigkeit ignoriert. (3) Mit der Überlastung von Ressourcen und Senken werden Lebensräume von Lebewesen vernichtet, die anders als der Mensch keine »exosomatischen Instrumente« haben ausbilden können, um sich eine $\gg$ zweite , an die veränderten Umweltbedingungen angepasste Natur zu formen. Wenn die Veränderung von »Umwelten« zu schnell erfolgt, als daß sich die innere Natur daran anpassen könnte, sterben die Arten aus »und zwar mit mindestens tausendfach höherer Rate als dies natürlicherweise jemals in den vergangenen $65 \mathrm{Mio}$. Jahren geschah « (Wissenschaftlicher Beirat: 7). Die Katastrophe besteht genau darin, daß die zeitlichen Spielräume - auch eine Folge des raum-zeitlichen Imperialismus, von Expansion und Beschleunigung - so eingeengt sind, daß Vorkehrungen zur Anpassung an eine radikal geänderte Umwelt unmöglich sind.

Die Übernutzung der globalen Öko-Systeme ist ein angesichts der in der besitzindividualistischen, kapitalistischen Gesellschaft geltenden »Regeln des Spiels« das Ergebnis der Verfolgung rationaler Interessen von Akteu- 
ren ${ }^{15}$. Das »Spiel《 kulminiert in der »tragedy of the commons« (Hardin 1968) ${ }^{16}$. Individuelle Rationalität kann nicht nur in kollektive Irrationalität umschlagen (ein Thema, dem sich die Sozialwissenschaften seit ihrem Entstehen widmen). Wegen der (positiven) Rückkopplungen mit anderen ebenso individuell und rational interessegeleiteten Akteuren und infolge des gemeinsamen Zugriffs auf begrenzte und sich daher für alle anderen quantitativ und qualitativ aufgrund individueller Aktionen ändernden Ressourcen und Senken (dadurch allein wird die Annahme von der Individualität des entscheidenden Akteurs fragwürdig) können die eigenen Interessen allenfalls vorübergehend realisiert werden - solange wie die Grenzen der Ressourcen nicht fühl- und wahrnehmbar sind, weil die Tragfähigkeit größer als die summierten Belastungen ist.

Dies alles setzt der entwicklungspolitisch angestrebten und durch die Konkurrenz erzwungenen ökonomischen Steigerung der Produktion doch wieder Grenzen, die eigentlich durch den Rückgriff auf die exosomatischen Energien überwunden werden sollten: Grenzen der Tragfähigkeit oder Belastbarkeit der globalen Ökosysteme, Grenzen der »ecological scale of production and consumption «. Die exosomatischen Apparate, eine Erleichterung und Vervielfachung der beschränkten endosomatischen Kräfte ihrer Anwender, werden zu einem Problem, weil sie sich verselbständigen wie der Besen des Zauberlehrlings. Die Logik der bisherigen Entwicklung folgte der »penetrative power of the price system «, der Dynamik des Geldes und der innovativen Potenzen des Marktes. Die aus ihr abgeleiteten Regeln und Handlungsmaximen sind ungeeignet, die Probleme der Übernutzung der »global commons « zu vermeiden oder, wenn sie denn eingetreten sind, Lösungen aufzuzeigen. Die modernen (kapitalistischen) Gesellschaften befinden sich vor der unerfreulichen Alternative, über ein ausgeklügeltes monetäres, ökonomisch höchst effizientes Steuerungssystem mit Marktpreisen zu verfügen, das aber - im Sinne Hardins (1968) - tragischerweise ungeeignet ist, Regeln für den Stoffwechsel mit der Natur und für die dabei erzeugten globalen ökologischen Probleme anzubieten. Je weiter das Programm der »Rationalität der Weltbeherrschung « verfolgt wird, desto mehr stellt sich die Unmöglichkeit heraus, die Welt wirklich beherrschen zu können: »a society that does not take into account the repercussions of its transformation of nature can hardly be said to dominate

15 Auch Regierungen können als individuelle Akteure, jedenfalls was den »output « des Regierungshandelns anbelangt, behandelt werden.

16 Elinor Ostrom (1990) beschreibt das Dilemma als einen speziellen Fall allgemeiner Problemlagen, in denen Individuen mit ihrer individuellen Rationalität versuchen (müssen), »collective benefits « zu erreichen. Die »tragedy of the commons《 ist daher strukturell vergleichbar mit dem spieltheoretischen »prisoner dilemma« oder der Olson'schen »logic of collective action (Ostrom 1990: \$. 2-28). 
nature at all« (Grundmann 1991, S. 109)17. Wenn es erstens Grenzen der Belastbarkeit der Ökosysteme auf regionaler und globaler Ebene gibt, wenn zweitens der »Rationalität der Weltbeherrschung « mit den Mitteln der kapitalistischen Produktionsweise folgend die Belastung der Ressourcen und Senken über die Grenzen der Belastbarkeit hinausschießt, wenn daher drittens ökologische Krisen unausweichlich sind, stellt sich viertens die Frage, wie der Stoffwechsel zwischen Gesellschaft und Natur auf globaler Ebene reguliert werden kann.

\section{Rationierung der Ansprüche an die Natur}

Prinzipiell gibt es zwei Antworten auf die Herausforderung begrenzter Ressourcen für den industriell-kapitalistischen Produktions- und Konsumtionsproze $\beta$ und überlasteter Senken für die Abgase, Abwässer und $\mathrm{Ab}$ fälle. Die eine Antwort lautet schlicht »containment« (Sachs 1992): Eindämmung der negativen Konsequenzen der Übernutzung von Ressourcen und Senken im »Süden « des Globus, in der »Dritten Welt«, um in den privilegierten Industrieländern des »Nordens« das eingeübte Produktionsmodell und den lieb gewordenen »life style « fortsetzen zu können. Die ökologischen Kosten der globalisierten Industriegesellschaft werden also auf einem Globus »externalisiert«, der gerade durch die zeitliche und räumliche Dynamik kapitalistischer Verwertungslogik immer $» k l e i n e r \ll$ wird und daher die Strategie einer Externalisierung inzwischen ausschließt. Die Betroffenheiten sind zwar ungleich verteilt - dazu später mehr -, aber es gibt auf der Erde kein Land, keine Region, die sich ignorant stellen könnte. Monetäre Kompensationen (etwa mit einer fiskalisch immer unzureichenden "Global Environmental Facility «) helfen nicht wesentlich weiter, sie sind eher Teil des zu lösenden Poblems. Das Modell einer gespaltenen Welt, einer »globalen Apartheid « durch Eindämmungsversuche, ist auf lange Sicht nicht nur ökologisch ausgeschlossen.

$»$ Containment« ist eine Strategie des Schlußstrichs: Sie erkennt an, daß raum-zeitliche Expansion an eherne Schranken stößt, so daß die Privilegierten ihre Claims gegen die anderen, die »nicht-saturierten « Nationen verteidigen müssen. Noam Chomsky zitiert in seinem Buch über die 500jährige Ordnung der kapitalistischen Welt Winston Churchill (Chomsky 1993, S. 9), der, ganz den Denkmustern der »westfälischen Ordnung « verhaftet, von der Gefahr »hungriger Nationen« eindeutig im

17 Ein Aspekt der Nicht-Beherrschung ist auch die Unmöglichkeit der ökologischen Kommunikation außerhalb der binären Marktcodes von Zahlen und Nichtzahlen (Luhmann 1990), die aber zur Erfassung der Transformationen von Stoffen und Energien im ökonomischen Prozeß unzureichend sind. 
Sinne der territorialen Nicht-Saturiertheit spricht und deren Eindämmung durch die bereits »saturierten Nationen « begründet. Containment heute gegen die »Nicht-Saturierten « hat inzwischen eine andere, eine meta-territoriale, ökologische Dimension erhalten. Der Unterschied zu den raum-zeitlichen Regimes der vergangenen Epochen kapitalistischer Entwicklung ist eklatant: Der Kolonialismus der Frühphase des Kapitalismus versuchte, die »weißen Flecken « zu erobern, zu unterwerfen und auszubeuten und subaltern, eben als Kolonien, in den »charmed circle« der kapitalistischen Nationalstaaten einzubeziehen, wobei sie nicht »zivilisiert « genug sind, um sich nicht doch in den kolonisierten Kontinenten ins Gehege zu kommen. Die imperialistischen Staaten des 19. und 20. Jahrhunderts unternahmen alle Anstrengungen, die bereits territorial aufgeteilte Welt räumlich zu reorganisieren - und sie gerieten bei diesem Unterfangen in kriegerische Konflikte, die sich zu Weltkriegen zuspitzten, da es ja um eine Neuordnung der Welt und um die Hegemonie ging. Es waren jeweils Nationalstaaten, die diese Versuche der territorialen (und staatsvölkischen, daher immer rassistisch begründeten) Ausdehnung der Macht unternahmen und so die »westfälische« Ordnung, die die Macht balancieren sollte, endgültig zum Scheitern brachten. Die Strategie der Eindämmung, also eine Strategie der ökonomischen, politischen, sozialen Abschottung und nicht der territorialen Ausdehnung, bringt eine gänzlich neue räumliche Organisation der Erde hervor. Sie ist den im Verlauf der Nachkriegsordnung entstandenen und in den Strukturen des internationalen System eingeschriebenen Privilegien nachgebildet. Die wohlständigen Gesellschaften versuchen die Zugriffsmöglichkeiten auf Ressourcen und Senken zu sichern, tragen aber bei den erkannten Grenzen der globalen Ökosysteme dafür Sorge, daß Einschränkungen vor allem bei anderen wirksam werden. Das Prinzip der Gleichheit von Ansprüchen an die Natur der Erde, von Bedürfnissen und von Möglichkeiten ihrer Befriedigung wird ersetzt durch eines der Rationierung der Nutzung begrenzter Ökosystem als Senken und Ressourcen. Ein kleiner Teil der Menschheit bekommt große, ein großer Teil der Menschheit bekommt kleine Rationen zugeteilt. Die Rationierung durch den Preismechanismus (jene G7-Bürger mit einem durchschnittlichen ProKopf-Einkommen von mehr als 20000 US\$ im Jahr können größere Rationen greifen als jene G77-Bürger Menschen aus der Gruppe der 77 Entwicklungsländer mit einem jährlichen Pro-Kopf-Einkommen von vielleicht 500 US\$) wird in der »neuen Weltordnung « mit politischen und militärischen Mitteln perfektioniert. Während die durch den Markt und den Preismechanismus besorgte Rationierung per se legitimiert scheint, bedüren politisches und militärisches Durchgreifen besonderer Legitimationsan- 
strengungen, zu denen auch die Versuche gehören, die UNO auf ein neues Fundament zu gründen.

Die Produktionsweise und der Lebensstil der Industriegesellschaft sind, weil auf dem Globus nicht von allen Menschen erreichbar, ein »oligarchisches Gut « (Harrod 1958; Hirsch 1980). Die Gleichheit der Möglichkeiten zur Befriedigung von durchaus ungleichen Ansprüchen von Menschen aller Kontinente, Kulturen und Rassen an die globalen Ressourcen und die Gerechtigkeit der Verteilung von Einkommen und Lebenschancen sind in der Industriegesellschaft paradoxerweise - denn »eigentlich « bietet ihr »Wohlstand« ungeheure Chancen der Um- und Gleichverteilung und der Entwicklung von humanen Fähigkeiten - ausgeschlossen. Eine »gerechte « Weltordnung auf der Grundlage des gegenwärtig in den Industrieländern im Exzeß vorgeführten Industriemodells gibt es nicht. Es gibt aber ebenfalls keinen sozialen Mechanismus, der bei Aufrechterhaltung der konstituierenden Prinzipien dieser Ökonomie und $\mathrm{Ge}$ sellschaft - monetäre Budgetrestriktion, Profitprinzip, Legitimationsbeschaffung aus individualisiertem Wohlstand, Konkurrenz - eine Beschränkung des Zugriffs auf die globalen Ressourcen vorsehen würde. Die ökologische Krise weist Ausmaße einer Zivilisationskrise auf (Anders 1980). Die Erhaltung der Ökosysteme kann nicht begründet werden, ohne an der Weise von Produktion und Konsumtion etwas zu ändern, die die Ungleichheit der Zugriffsmöglichkeiten auf Natur konstituiert hat. Wenn also die ökologische Degradation auf Erden beklagt wird und gleichzeitig die tragenden Prinzipien der Weltordnung »am Ende der Geschichte«, Markt, Kapitalakkumulation und parlamentarische Demokratie für tabu erklärt werden, bekommen die ökologischen Argumente einen konservativen und autoritären Hang, der vielen ökologischen Diskursen eigen ist 18 . Umgekehrt aber zeigt es sich, daß ökologische Diskurse des Bezugs auf Normen sozialer Gerechtigkeit bedürfen. Denn wenn Rationierung der Ansprüche an die Natur unvermeidbar ist und wenn diese dem Preismechanismus des Marktes aus guten Gründen nicht überlassen werden kann, müssen Kriterien aus einer Theorie der ökologischen Gerechtigkeit abgeleitet werden, die es freilich allenfalls in Ansätzen gibt (vgl. Ely 1989).

\section{7. "Sustainability - ein falsches Versprechen?}

Kommen wir zur Möglichkeit einer anderen Antwort auf die ökologische Herausforderung. Vorstellbar wäre eine Art Gleichverteilung von Entnahmerechten an den Ressourcen der Erde und von Belastungsrechten an den

18 Es ist nicht möglich, die konservativen und rechten ökologischen Diskurse an dieser Stelle adäquat zu referieren. Vgl. dazu Jahn/Wehling (1991). 
globalen Schadstoffsenken. In diesem Sinne wird die UNCED-Konferenz von Rio de Janeiro im Juni 1992 als bedeutender Beitrag zur Regimebildung interpretiert (Bruckmeier 1994; Simonis 1993; Loske 1993; Rowlands 1992), zumal der UNCED-Konferenz andere Abkommen zur globalen Regulation des Stoffwechsels mit der Natur vorausgegangen sind. Sie zeichnet eines aus: Eine Art »conditionality «, die das Prinzip des unbedingten $»$ free trade « und der »freien Unternehmerinitiative « gewissermaßen an eine (allerdings sehr lange) Leine legt, ohne dem Prinzip des nationalstaatlichen Protektionismus (dieses Prinzip stammt aus der Weltordnung der Nationalstaaten des 19. Jahrhunderts, auch wenn es heute nicht ganz unaktuell ist) das Wort zu reden. Dazu gehören das Abkommen über den Schutz von seltenen Tieren und Pflanzen (Washington Agreement) von 1975, die FCKW-Konvention von Wien (1985) und Montreal (1987) mit den Nachfolgevereinbarungen von London und Kopenhagen (Vgl. Wissenschaftlicher Beirat 1993: 20ff.), das Baseler Müllabkommen von 1989, das im März 1994 in Genf beträchtlich verschärft worden ist, die Tropical Timber Trade Organization von Tokio und Yokohama, und schließlich die in Rio beschlossene, inzwischen ratifizierte $\mathrm{CO}_{2}$-Convention sowie die weniger verbindlichen Abkommen über den Schutz der Wälder und der Artenvielfalt, ebenfalls von Rio de Janeiro. Es wäre übertrieben, diese und die anderen etwa 170 umweltrelevanten internationalen Abkommen (French 1993; Petersmann 1992; OECD 1991, S. 281ff, wo eine Liste der Abkommen $z u$ finden ist) bereits als $\gg$ Regime $\ll$ mit den oben genannten Elementen zu interpretieren, das Rahmen, Ziele und Wege des umweltpolitischen Handelns von internationalen Akteuren umschreiben würde. Das Novum der Bildung eines internationalen Umweltregimes besteht darin, daß die Regulation des Stoffwechsels mit der Natur nicht mehr allein der Preissprache des Weltmarkts, aber auch nicht mehr den selbstverständlichen Kompetenzen von doch nicht souveränen Nationalstaaten überlassen bleibt, und Elemente des ökologischen Ausgleichs zwischen Gesellschaften ansatzweise enthält. Das Schema indiziert diesen Zusammenhang.

Woran können sich politisch gesetzte (also nicht mehr allein durch Preise definierte) Grenzen der Nutzung von globalen Ressourcen und Senken orientieren, wo sind sie zu markieren, zu etablieren und geflissentlich zu kontrollieren? Grenzen auf räumlichem Territorium zu setzen und zu kontrollieren, ist Privileg des souveränen Nationalstaates. Aber »sein« eingegrenztes und eingefriedetes Territorium ist ja mannigfachen, nicht zuletzt ökologischen Einwirkungen ausgesetzt. Was sind die Grenzen des grenzenlos kosmopolitischen Freihandels? Sie sind jene der »Budgetrestriktion des Geldes «, also eine nicht-territorial-räumliche, eine (ökonomisch) funktionsräumliche Grenze (zu dieser Unterscheidung vgl. Altvater 1987): Nur 
die Handelstransaktionen sind durchführbar, die gemessen an den internationalen Zinssätzen rentabel sind. Die Grenzen der Nutzung von ökologischen Ressourcen, beispielsweise des Eintrags von Schadstoffen in die Atmosphäre, können nicht in nationalstaatlicher Abgrenzung definiert oder der ökonomischen Budgetrestriktion unterworfen werden. Versuche, die in diese Richtung weisen, scheitern entweder daran, daß sie sich territorial nicht eingrenzen lassen, oder daran, daß die Verfolgung des Rentabilitätsund Profitprinzips gerade die Externalisierung veranlaßt. Oder die interne Grenzziehung im Zuge der »Zonierung《 eines Territoriums gemäß ökonomischen, ökologischen, militärischen, ethnisch reservierten Nutzungsrechten scheitert an der Inkompatibilität von territorial-räumlich orientierten und ökonomischen Interessen einerseits und der Logik der Beschaffung politischer Legitimation andererseits (dazu: Nitsch 1993).

Regulation ökologischer Standards; Ansätze der internationalen Regimebildung

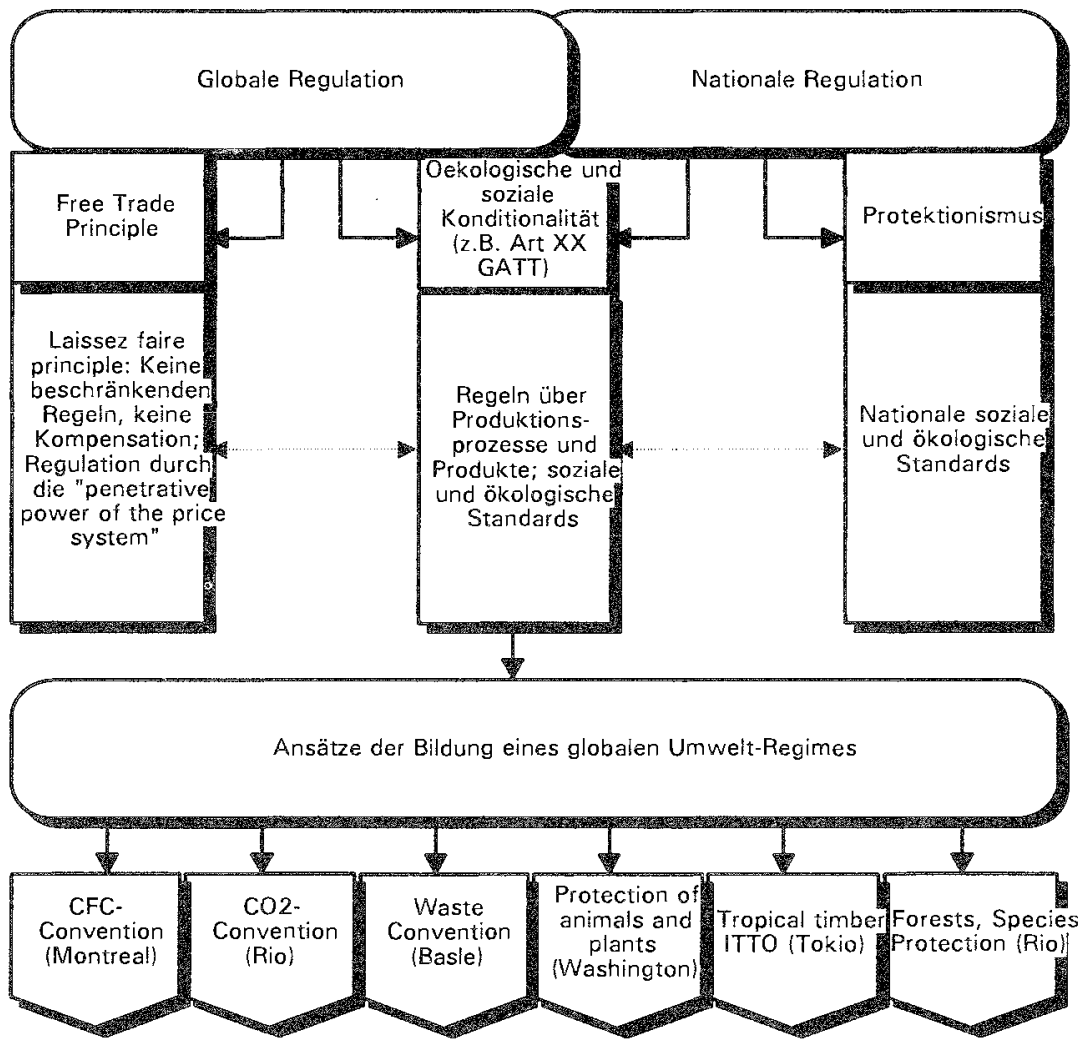


Vielmehr sind ökologische »Budget«restriktionen doppelt bestimmt. Erstens - passiv - durch die Tragfähigkeit und Belastbarkeit der globalen Ökosysteme im Hinblick auf anthropomorphe Einwirkungen und zweitens - aktiv - durch das Ausmaß von Belastungen, die im Prozeß von Produktion und Konsumtion durch Menschen auf dem Erdball erzeugt werden. Diese neue Grenze zwischen ökologischer Belastbarbeit und ökonomischer Belastung, zwischen »ecological scale« und »economic scale« von Produktion und Akkumulation wird in der internationalen Debatte, insbesondere seit Erscheinen des Brundtland-Berichts 1987 (Hauff 1987), mit dem Begriff der »sustainability « zu markieren versucht. Der Begriff ist normativ aufgeladen und analytisch wenig stringent ${ }^{19}$. Sinnvollerweise muß er an den oben bezeichneten globalen ökologischen Problemen anknüpfen und Gestaltungen des Stoffwechsels zwischen Mensch und Natur begründen, deren $M a \beta$ die Fähigkeit zur Reproduktion und Evolution der Arten ist: Weder dürfen Ressourcen und Senken über die Regenerationsfähigkeit hinaus genutzt, noch darf die Evolution der Arten den Interessen der Geldvermögens- (und Genbanken)besitzern überantwortet, also der ökonomischen Budgetrestriktion und der ihr unterworfenen Logik des Handelns ökonomischer Agenten ausgesetzt werden. Dabei könnte nur eine hochentropische Monokultur herauskommen, deren (Über)lebensfähigkeit fraglich ist. Also wäre »Nachhaltigkeit« sinnvollerweise thermodynamisch zu definieren (Daly 1991; Goodland 1992; Altvater 1992): Die (ökologische) Entropieproduktionsrate muß auf der Erde $=0$ gesetzt werden, d. h. Energiezufuhr (von der Sonne) und Entropiezunahme in Form von Abwärme, Abwasser, Müll, Abluft etc. müssen sich die Waage halten (Georgescu-Roegen 1971; Daly 1991). Die Zielgröße eines globalen Umweltregimes ist also bezeichnet, doch wie soll das Regime operieren?

Das Prinzip der Nachhaltigkeit kann auf zwei Wegen angestrebt werden: Die Nutzung von Ökosystemen hängt ja erstens davon $a b$, wieviele Ressourcen entnommen und wieviel Emissionen in die natürlichen Senken eingetragen werden, also von der »ecological scale of production and consumption « (Zarsky 1993). Dies ist die stofflich-energetische, die gebrauchswertmäßige Seite von Produktion und Konsumtion. Zweitens wird das Ausmaß der Ressourcennutzung von dem Niveau, der Wachstumsrate und der Verteilung des Einkommens in der Weltgesellschaft, von der

19 Obendrein ist er sehr bequem, da er den Eindruck von »win-win«-Strategien vermittelt: Unter diesem begrifflichen Dach »gewinnen« die Entwicklungsstrategen ebenso wie die Umweltschützer. Der Begriff legt Synthetisierung nahe, bevor die Widersprüche zwischen Umwelt und Entwicklung analytisch ausgelotet worden sind. Daher ist die Literatur zur »Nachhaltigkeit« so unbefriedigend, ihr mangelt die notwendige Schärfe (vgl. als Beispiel die meisten Beiträge in Hein 1993). 
»economic scale of production and consumption « bestimmt. Dabei handelt es sich um die wertmäßige Seite ökonomischer Prozesse (dazu in Anknüpfung an der Marxschen Kategorie des »Doppelcharakters « von Arbeit und Produktion: Altvater 1991 und 1992). Die Regeln eines internationalen Regimes können nun an der einen oder der anderen »scale « ansetzen oder an beiden. Die Nutzung von Ressourcen und Senken (ecological scale) verlangt die Setzung verbindlicher quantitativer Höchstwerte und qualitativer Standards, beispielsweise über zulässige $\mathrm{CO}_{2}$-Emissionen, die Art und Weise der Waldnutzung, die Gewässerbelastung, die Produktion und Behandlung von Müll einschließlilch der Maßnahmen zum Recycling etc. Verbindliche Obergrenzen der Ressourcenbeanspruchung erfordern einen politischen Konsens über Grenzwerte, daraus abgeleitete Verbote und Gebote und die Art und Weise ihrer wirksamen Durchsetzung und Sanktionierung. Dieses Ziel zu erreichen ist nicht nur technisch äußerst schwierig (dariiber gibt die »Grenzwertdebatte « Auskunft), sondern sozial utopisch. Und dennoch muß es angestrebt werden, da es keine andere Alternative zur globalen ökologischen Degradation gibt.

Die Beachtung der Grenzen der »economic scale« hingegen kann im Prinzip mit ökonomischen Methoden durchgesetzt werden: Die Akteure werden veranlaßt, Umweltbelastungen, die sie verursachen, zu internalisieren. Dazu können einerseits Umweltsteuern und die Streichung aller umweltbelastenden Subventionen (im Transportsektor, in der Landwirtschaft, in der Energieproduktion) sowie eine angemessene Gestaltung öffentlicher Tarife eingesetzt werden. Andererseits kann mit Regeln der Preisbildung seitens der Unternehmen die »Preiswahrheit « gesteigert werden. Bislang externalisierte Kosten müssen, so die Regel, internalisiert werden. Marktliberale Vertreter (aber auch die Ökonomen der Weltbank) lieben die Vorstellung »ökologisch richtiger Preise«, weil sie so im vertrauten marktwirtschaftlichen Diskurs verbleiben können, und sie gehen davon aus, daß eine Erhöhung der Allokationseffizienz durch Internalisierung der (externalisierten) Kosten der Umweltbelastung in die Preiskalkulation eine Reduzierung von Ressourcenverbrauch und Schadstoffeintrag herbeiführen könnten (ein gegebenes Einkommensniveau vorausgesetzt). Allerdings sind ernsthafte Zweifel an den Erwartungen, mit »richtigen Preisen« die Umwelt entlasten zu können, nicht nur deshalb anzumelden, weil empirische Untersuchungen beispielsweise über »structural adjustment loans« der Welṫbank ergeben haben, daß die »conditionality«, zu der immer auch die Regel »getting the prices right « gehört, zwar die Allokationseffizienz verbessern und daher die »economic scale« der ökologischen Belastung reduzieren kann, aber auf die »ecological scale« nur unzureichend einwirkt (Zarsky 1993). Mit Regeln, die sich an der ökonomischen Budgetrestrik- 
tion orientieren - ganz abgesehen von jenen, die die (äußeren und inneren) politischen Grenzen des Territoriums zum Kriterium machen -, lassen sich die ökologischen Restriktionen nicht sinnvoll bearbeiten.

\section{Die Illusion »richtiger Preise«}

Dafür ist eine Reihe von Gründen maßgebend. Erstens ist eine vollständige Internalisierung externer (ökologischer) Kosten ja schon aus Gründen, die zur Formulierung des 2. Hauptsatzes der Thermodynamik geführt haben, ausgeschlossen: Stoffliche und energetische Transformationsprozesse sind irreversibel und hinterlassen dissipative Strukturen, die auch bei vollständiger Kostenkalkulation nicht revidiert werden können. Die Preise mögen im Idealfall zwar richtig sein. Die Umweltzerstörung wird dadurch aber nur kalkulatorisch erfaßt, nicht aber uno actu und realiter verhindert oder rückgängig gemacht. Das Problem wird noch dadurch gesteigert, daß Umfang, Wirkungsgrad und Wirkungsrichtung stofflicher und energetischer Transformationen prinzipiell nicht vollständig bekannt sein, also auch nicht in »richtigen Preisen« kalkuliert werden können.

Ein zweiter Grund hat damit zu tun, daß die Marktpreisbildung unter den obwaltenden Marktbedingungen gar nicht zu »richtigen« Preisrelationen führen kann. So sind mindestens $25 \%$ des Welthandels »intra-firm trade «. Die Preise auf dem Weltmarkt sind daher gar nicht Resultat des freien Spiels der Kräfte, sondern Ergebnis mikroökonomischer Preissetzungsmacht des Managements von transnationalen Unternehmen (OECD 1993). Obendrein ist drittens der zentrale, weil die Budgetrestriktion des Geldes ausübende Preis einer kapitalistischen Geldwirtschaft, der Zins nämlich, eine höchst unzuverlässige Variable insbesondere in Zeiten zugespitzter ökonomischer Instabilität. Seine Höhe reflektiert nicht mehr - wie die Klassiker annahmen und Knut Wicksell zum Theorem erhob - die realen und »natürlichen« Möglichkeiten der Erzielung eines Überschusses (indiziert vom Anstieg der Produktivität) und daher eines Profits auf das in die Produktion investierte Kapital. Infolge des globalen »debt overhang « und der Internationalität des Kredits bei gleichzeitiger Nationalität von Währungen - dies ist ein permanenter Anlaß für Spekulationen - wird im Zins vor allem das Risiko von Kapitalausleihungen und -investitionen abgegolten. Der mit einer bestimmten Kapitalsumme erzielbare produktive Überschuß in Form des Profits ist für die Renditekalkulationen der innovativen »Finanzinstrumente« auf dem monetären Weltmarkt unbedeutend. Wie in der "prä-fossilistischen « Zeit des kanonischen und islamischen Zinsverbots ist die Höhe des Zinses von der realen (und sozialen) Leistungsfähigkeit von Schuldnern abgekoppelt. Da es sich in der gegenwärti- 
gen Welt zu einem Gutteil um souveräne Schuldner (Staaten) handelt, werden in der Folge politisch moderierte Umverteilungsprozesse zu Gunsten der internationalen Geldvermögensbesitzer ausgelöst. »Moderatoren « sind dabei vor allem die Weltbank, der Internationale Währungsfonds, die Clubs von London und Paris, die Bank für internationalen Zahlungsausgleich in Basel (BIZ) etc. - alle jene Institutionen also, die im Zuge der Nachkriegs-Weltordnung gegründet oder - wie die BIZ - weiterentwickelt wurden und zwischenzeitlich einen Funktionswandel haben durchmachen müssen. Die realen Nettotransfers aus den verschuldeten Ländern des »Südens « an die Gläubigerbanken in den »nördlichen« Ländern unterstreichen diese Tendenz. In vielen Gesellschaften wird die soziale und die ökologische Substanz geschädigt; darüber hinaus werden beträchtliche Teile der Bevölkerung in die Subsistenzproduktion und in einen informellen Sektor prekärer Beschäftigung abgedrängt. Die Bildung des Zinssatzes ist zwar ökonomisch rational, der Preis ist als hoch reagibler Marktpreis theoretisch so »richtig «, daß Ökonomen eine politische Regulation von Preisbildungsprozessen auf internationalen Kapital- und Geldmärkten heftig ablehnen (z. B. Dornbusch 1994). Wenn aber die Höhe des Zinses sich von den realen Bedingungen der Überschußproduktion löst und vor allem die Höhe des Risikos von Ausleihungen reflektiert, kann er nicht ökologisch und sozial »richtig « sein und rationale Entscheidungen anleiten.

Kann viertens die Limitierung der »economic scale« durch eine Art »ecological conditionality « im internationalen Handel erfolgen? Die Entscheidung für eine ökologische Konditionalität, um die kein Weg herumführt, sofern die UNCED-Beschlüsse von Rio und danach ernst genommen werden, zieht eine Alternative nach sich: Entweder liegt die Kontrolle der ökologischen Normen bei den Nationalstaaten oder sie werden in einem internationalen Handelsabkommen verankert und von einer internationalen Institution überwacht. Im ersten Fall sind »Öko-Dumping« auf der einen und »Öko-Protektionismus« auf der anderen Seite kaum zu vermeiden und zwischenstaatliche Handelskonflikte sind schon jetzt vorgezeichnet. Diese Perspektive muß nicht schrecken, zumal die Alternative des ökologisch nicht regulierten freien Handels keineswegs freundlicher ist. Die von den Klassikern versprochenen »komparativen Kostenvorteile« sind bei höchst mobilem Kapital und transnationaler Migration von Arbeitskräften noch nicht einmal theoretisch zu begründen (Daly 1994). Vorzuziehen wäre freilich die Errichtung einer internationalen Institution, die die Regimebildung nach UNCED einen Schritt vorwärts bringen könnte ${ }^{20}$. In diese

20 Bei Abschluß des Manuskripts wurde gerade in Marrakesch die Welthandelsorganisation aus der Taufe gehoben, die sich gemäß der Absichtserklärung auch mit der Erarbeitung von Umweltstandards im Welthandel befassen wird. 
Richtung weisen Diskussionen über ein »greening of the GATT « (Anderson/Blackhurst 1992). Eine ökologische Konditionalität würde auf jeden Fall mit dem Prinzip des Freihandels, mit Nicht-Diskriminierung und Meistbegünstigung brechen und so die tragenden Säulen des GATT unterminieren ${ }^{21}$. Da es im Falle der Einrichtung einer ökologischen Konditionalität immer um begrenzte Nutzungsrechte an der Natur geht, die für die Erhaltung von Einkommensniveaus unabdingbar sind, da obendrein finanzielle Beiträge zu leisten sind, ist Regimebildung notwendigerweise mit Verteilungskonflikten über Transfer- und Kompensationszahlungen verknüpft. Sollte etwa eine Energie- oder $\mathrm{CO}_{2}$-Steuer erhoben werden, stellen sich sofort die Fragen nach der Steuerinzidenz (müssen die Konsumenten von Energiedienstleistungen sie tragen oder die Produzenten der Nutzenergie oder die Erdölproduzenten als Erzeuger des Energieträgers) und der Budgetwirkung der Steuer in den Industrieländern, bei internationalen Institutionen, in Entwicklungs- oder Erdöl produzierenden Ländern (Whalley/Wigle 1991).

\section{Akteure im »globalen Dorf « oder: ein Schritt zu einer transnationalen Zivilgesellschaft}

Auch wenn wir gewohnt sind, von »globaler Ökologie«, von einem »globalen Dorf« (McLuhan) oder der »Einen Welt« zu sprechen, wenn wir »global denken « und »lokal handeln «, besteht das Besondere des internationalen Systems doch gerade darin, daß Verursacher und Betroffene, Umweltschädiger und -geschädigte noch weniger miteinander zu tun haben, wenn eine nationale Grenze das Territorium durchschneidet, als dies in der Region oder innerhalb einer Nation der Fall ist. Daher ist politische Kooperation in der internationalen Öko-Diplomatie noch schwieriger zu erreichen als in vergleichsweise homogenen und kohärenten Einheiten, wo die Herstellung eines Konsenses schon schwierig genug ist (Ostrom 1990; Streeten 1992).

Entgegen den Theorien der internationalen Beziehungen, insbesondere wenn die »realistischen« Beiträge herangezogen werden, sind die Nationalstaaten nicht mehr die einzigen, und in mancher Hinsicht möglicherweise auch nicht mehr die ausschlaggebenden Akteure in einem letztlich anarchi-

21 »The GATT, with its present thirty-eight articles, makes no explicit reference to environmental matters. Thus, trade measures justified on arguments about unfair trade linked to environmental considerations are unlikely to have a foundation under GATT. They would almost certainly be ruled as GATT-incompatible by GATT panels if complaints were brought « (Whalley 1991: 181). Eine sehr viel positivere Interpretation der Spielräume des GATT-Abkommens gibt Petersmann 1992: 262f. Vgl. auch Altmann 1992 und Kulessa 1992. 
schen internationalen System, auch wenn die kulturellen, sprachlichen, politisch-historischen Traditionen den staatlich vermittelten Umverteilungsmaßnahmen eher die benötigte Legitimation verleihen als Appelle an kosmopolitische Gemeinsamkeiten von Weltbürgern. Auch die gesteigerte Bedeutung von Nationalstaaten in der internationalen Standortkonkurrenz ist eher Audruck von Souveränitätsverlust als von nationalstaatlicher Potenz, da es doch nur um die Vornahme von Anpassungsmaßnahmen an die Weltmarktbedingungen durch den $»$ Wettbewerbsstaat« geht. Neben Staatengruppen, wie der großen und mächtigen Europäischen Union oder der kleinen und schwachen $\gg$ Alliance of Small Island States« (AOSIS), neben losen Allianzen wie die Gruppe der 77 und fest institutionalisierten Koordinierungsgremien wie der Gruppe der 7 treten in der internationalen Arena transnationale Unternehmen und Banken mit ihrer in politische Macht umgesetzten ökonomischen Potenz auf. Es sind internationale Institutionen wie die Weltbank oder der Internationale Währungsfonds, das GATT oder die ILO, häufig als Verstärker der ökonomischen und politischen Macht der Industrieländer präsent, und es wirken Nicht-Regierungsorganisationen im Prozeß der Entscheidung insbesondere in den Umwelt und Entwickung betreffenden Fragenkomplexen mit. Dazu gehören so heterogene Akteure wie jene finanzkräftigen, auf internationalem Parkett routinierten und gut ausgerüsteten Umweltschutz-Organisationen aus dem »Norden« (Greenpeace; Friends of the Earth; Conservation International etc.) und Repräsentanten der »ecosystem peoples «, der indigenen Völker aus den Regenwaldgebieten (z. B. Aliança dos povos da floresta aus Amazonien), die weder über große Mittel noch über Erfahrungen verfügen (vgl. Rowlands 1992). Die erwähnten Akteure verfolgen Interessen. Diese können nach Verursacher- und Betroffeneninteressen untergliedert werden. Als Zwischenkategorie werden noch die von Prittwitz (1990) so genannten »Helferinteressen« berücksichtigt. Aus der so entstehenden Matrix von Akteuren und Rollenverteilung kann man bereits einen Eindruck von der Komplexität und Widersprüchlichkeit der Interessenstruktur auf internationaler Ebene gewinnen, wenn es um die Aushandlung von Regimen geht, die Entwicklung und Umwelt regulieren sollen. Die Bildung eines Umwelt- und Entwicklungsregimes ist ebenso schwierig wie die Regulation der Nutzung von Ressourcen und Senken durch die »penetrative power of the price system « leicht ist - in der Wirkungsweise, nicht hinsichtlich des Ergebnisses. Das folgende Schaubild deutet diese Zusammenhänge an, es knüpft an einer Darstellung von Simonis (1993) an und führt sie weiter. 
Akteure und deren Interessen in der globalen Umweltpolitik

\begin{tabular}{|c|c|c|c|c|}
\hline $\begin{array}{l}\text { Internationale } \\
\text { Akteure }\end{array}$ & $\begin{array}{l}\text { Verursacher } v . \\
\text { Emissionen }\end{array}$ & $\begin{array}{l}\text { Betroffene } \\
\text { von } \\
\text { Emissionen }\end{array}$ & $\begin{array}{l}\text { Geber von } \\
\text { Hilfe }\end{array}$ & $\begin{array}{l}\text { Empfänger von } \\
\text { Hilfe }\end{array}$ \\
\hline \multicolumn{5}{|l|}{$\begin{array}{l}\text { Staaten und } \\
\text { Staatengruppen }\end{array}$} \\
\hline USA & stark & schwach & stark & kein Problem \\
\hline$E U$ & stark & stark & stark & kein Problem \\
\hline Osteuropa & stark & stark & schwach & stark \\
\hline OPEC & stark & schwach & schwach & schwach \\
\hline Gruppe 77 & schwach & slark & schwach & stark \\
\hline AOSIS & $\begin{array}{r}\text { weniger als } \\
\text { schwach }\end{array}$ & sehr stark & $\begin{array}{r}\text { kein } \\
\text { Problem }\end{array}$ & schwach \\
\hline $\begin{array}{l}\text { Transnationale } \\
\text { Unternehmen }\end{array}$ & stark & stark/schwach & $\begin{array}{r}\text { kein } \\
\text { Problem }\end{array}$ & kein Problem \\
\hline $\begin{array}{l}\text { Nicht- } \\
\text { Regierungs- } \\
\text { Organisationen }\end{array}$ & kein Problem & stark & stark & stark \\
\hline $\begin{array}{l}\text { Internationale } \\
\text { Institutionen } \\
\text { (IWF etc.) }\end{array}$ & kein Problem & kein Problem & stark & kein Problem \\
\hline
\end{tabular}

Das Schaubild ist nur die Momentaufnahme eines dynamischen Prozesses. Die Zuordnung von Interessen zu Staaten kann sehr schnell obsolet werden, wenn ein Regierungswechsel stattfindet. Dafür ist der Übergang von der Bush- zur Clinton-Regierung in den USA ein Beispiel, das für eine beträchtliche Änderung der umwelt- und entwicklungspolitischen Leitlinien steht. Die Frage ist, wie diese Dynamik zwischen globalen, nationalen und regionalen Prozessen, zwischen ökologischen Zielsetzungen und sozialen Interessen zustandekommt und wodurch sie beeinflußt werden kann. Abgesehen von ökonomischen Interessen und Einflüssen, kulturellen Traditionen und »Bildern ${ }^{22}$, der Erbschaft einer spezifischen Klassenstruktur, die

22 Es ist von größter Bedeutung, wje der in einer Waldkonvention zu schützende »Wald« gesehen wird, als der »böse, finstere Wald « in Dantes Inforno, als Holzressource der forstwirtschaftlichen Literatur, als Lebensraum scheuer Tiere, als »Hollywood《 und DinoDschungel, als »grüne Hölle« der Abenteuerromane... 
eine eigentümliche inner-nationalstaatliche Dynamik mit Auswirkungen in der internationalen Akteursarena erzeugen, muß auf die besondere Rolle der Nicht-Regierungsorganisationen in diesem Zusammenhang verwiesen werden. Die "neue Weltordnung « ist keine reine (National)Staatenordnung mehr. Infolge der globalen Kommunikation und Vernetzung hat der Staat, haben die Diplomaten von Regierungen nicht mehr das selbstverständliche und unangreifbare Monopol der Gestaltung aller Aspekte internationaler Beziehungen. Die $\gg$ Zivilgesellschaft $\ll$ ist dabei, sich zu trans- und zu internationalisieren. Auch dies ist eine Folge der Globalisierung des fossilistisch-fordistischen Modells, die keineswegs in Harmonie und ohne Konflikte zustandekommt. So entstehen auf der einen Seite wegen der Bedrohung der natürlichen Umwelt auf Erden die »neuen Betroffenheiten« und auf der anderen Seite die technisch möglich gewordenen internationalen Vernetzungen, die mehr und mehr organisatorische Form gewinnen, und zwar auf dem jeweils aktuellen technischen Niveau von globaler Kommunikation. Nicht-Regierungsorganisationen haben bei der Aushandlung internationaler Abkommen, insbesondere im Bereich von Umwelt und Entwicklung, inzwischen wichtige Aufgaben übernommen. Damit wird jenseits aller praktischen Fragen ein staatstheoretisches Problem aufgeworfen. Im internationalen System sind die Akteure mit längster Tradition, größter Machtausstattung, unbefragter Legitimation, größter Expertise jene souveränen Staaten, die seit der Heraufkunft der Moderne die internationale »Ordnung« bilden, von der zu Beginn die Rede war. Die Souveränität des Nationalstaats ist doppelt definiert und gleichzeitig begrenzt. Die Staatsmacht bezieht sich auf ein Territorium, hat also eine territoriale, räumliche Dimension, und sie leitet sich aus dem Staatsvolk her, das als Souverän in demokratischen Systemen die jeweilige nationale Regierung mit legitimierten Handlungsvollmachten ausstattet ${ }^{23}$. Wie auch immer hergeleitet und definiert - beide Ressourcen von Macht und Souveränität, Territorium und Staatsvolk, unterliegen einem Prozeß der Veränderung.

Die Grenzen des Nationalstats des 19. und 20. Jahrhunderts sind immer weniger kompatibel mit der Reichweite der ökonomischen Prozesse und mit den räumlichen und zeitlichen ökologischen Folgen von Stoff- und Energietransformationen. Die Souveränität von Staaten besteht, wie Poulantzas ausführte (1978), im Prinzip darin, Grenzen sowohl nach außen wie nach innen zu setzen, also ein geographisches und »funktionsräumliches« Territorium abzugrenzen, das freilich in Auflösung begriffen ist: Aus dem

23 Dabei darf freilich nicht übersehen werden, daß sich Nationalstaaten ihren Souverän konstruieren, etwa in Deutschland mit dem ius sanguinis, das kulturell adaptierte Bürger als Staatsbürger ausschließt und kulturell und sozial Fremde zu Staatsbürgern deklariert, wenn sie »deutsches Blut« nachweisen können. 
Prinzip »cuius regio, eius religio«, mit dem nach der Reformation die Religionswahl zwischen Protestantismus und Katholizismus in die Hand des jeweiligen regionalen Herrschers gelegt war und das ein Element der »westfälischen Ordnung« wurde, kann eine Regel »cuius regio, eius economia« (Schmitt 1974, S. 226, zit. nach Heins 1994, S. 79) nicht mehr abgeleitet werden. Sie wirkt eher lächerlich angesichts von Währungskonvertibilität, Welthandel und transnationaler Kapitalbewegungen. Infolge der Internationalisierung der Ökonomie ist das politische System der modernen Nationalstaaten »fluid« (Ruggie 1993, S. 139) geworden. Mit der tendenziellen $\gg$ Deregulierung « im Zuge der »neoliberalen Konterrevolution« ist mit der Absage an politische Regulation ökonomischer und sozialer Prozesse auch ein Stück politischer Souveränität im globalen Raum der ökonomischen Sachzwänge »verflüssigt« worden. Der »Wettbewerbsstaat « ist weder interventionistisch noch »Nachtwächter«, sondern in höchst widerspriichlicher Weise zugleich der »Virtualität « der globalen Finanzmärkte und Kommunikationsnetzwerke ausgeliefert und auf die $\gg$ Realität« von »Standorten« bezogen, die an die »Sachzwänge« durch Sicherung von Wettbewerbsfähigkeit ständig angepaßt werden müssen (vgl. Hirsch 1994). Die Souveränität von Nationalstaaten besteht in der Einsicht der politischen Klasse in die Weltmarktzwänge und in der Fähigkeit, ihnen mit einer Wettbewerbsstrategie Folge zu leisten.

Nun kann aus dieser Tendenz keineswegs geschlußfolgert werden, daß der Nationalstaat seine einstmals historisch entscheidende Rolle in der zweiten Hälfte des 20. Jahrhunderts vollends ausgespielt habe. Die Beschaffung von Legitimation für Regierungshandeln erfolgt immer noch in erster Linie in der durch die Nationalität definierten Gesellschaft, durch das Wahlvolk. Die Territorialität des Nationalstaats wird »entbündelt «, wie Ruggie (1993) den Prozeß der Erosion des nationalstaatlichen Territoriums als funktionaler Einheit und die Auflösung eines nationalen Territoriums in der Welt der sozialen Vorstellungen und Bilder (im »l'imaginaire social«) bezeichnet. Auch das Staatsvolk ist keine selbstverständliche Einheit mehr, wenn es das denn je war. Zugleich bilden sich subnationale, regionalistische Einheiten der Privilegierten heraus. Das führt zum Entzug von Ressourcen, aus denen der Nationalstaat seine Legitimation bezieht. Der selbst- und nationalbewußte Staatsbürger wandelt sich mit diesen Änderungen ebenfalls. Wenn sich sein tradiertes Aktionsfeld auflöst, die Symbolik seines Selbstbewußtseins nach und nach mit der nationalen Staatsmacht schwindet, transzendiert der (National)staatsbüger in einen trans- und international bewußten, weltoffenen Bürger oder er mutiert in den in Europa inzwischen wohlbekannten nationalistischen und regiozentrischen »Wohlstandschauvinisten« (Habermas). 
Dennoch: Alle diese Tendenzen haben die Nationalstaaten nicht von der Bühne getrieben, sie haben nur die Regeln des Spiels verändert und weitere Akteure auf den Plan gerufen. Denn nicht zuletzt sind Nationen als Währungsräume paradoxerweise international und zugleich national-ökonomisch definiert. Sie sind durch Wechselkurse gegeneinander abgegrenzt und mit der Zahlungsbilanz aufeinander als Nationalstaaten bezogen. Allerdings sind die Wechselkurse nur bedingt in der Gestaltungsmacht nationaler Staaten oder internationaler Institutionen. Sie sind das Resultat des »freien Spiels« der monetären Kräfte auf Devisenmärkten, auf denen heute täglich $1000 \mathrm{Mrd}$. US\$ umgesetzt werden - davon werden allenfalls 10 Mrd US\$, also rund 1\%, zur Abwicklung des Welthandels (Die Weltexporte betragen rund $3600 \mathrm{Mrd}$ US\$ pro Jahr) benötigt. Der Rest ist Spekulation, die wegen der nationalen Verfaßtheit der Währungsräume bei Globalisierung der Kapitalbeziehungen eine Notwendigkeit der Absicherung des Werts von Geldvermögen gegen Wechselkursbewegungen ist und gleichzeitig die »volatility « der Kurse erhöht.

Die »Ungleichzeitigkeitslücke « zwischen nationalstaatlicher Verfaßtheit, der Herausbildung eines internationalen ökonomischen und politischen Systems und der Globalität der ökonomischen, sozialen und ökologischen Probleme kann prinzipiell auf zwei Weisen geschlossen werden. Aus der Globalisierung der Ökonomie und der ökologischen Problemlagen sowie ihres Regelungsbedarfs ließe sich die funktionale Notwendigkeit globalisierter politischer Systeme der Regulation begründen (Knieper 1991 und 1993) bis zur Bildung eines globalen Staates. Dieser dürfte aber eher mit geringer Kapazität, die Probleme von Weltmarkt und globaler Ökologie lösen zu können, ausgestattet sein und würde obendrein, möglicherweise aber gerade aus diesem Grunde zu einem autoritären Alptraum degenerieren. Inwieweit ein globaler Staat überhaupt »personified, symbolized, imagined « (Walzer 1967, 194) werden und so als Staat einer globalen Gesellschaft Legitimation erwerben und auf Basiskonsens zählen kann, sei erst recht dahingestellt. Die von den »Realisten« unter den Theoretikern der internationalen Beziehungen beschworene Anarchie wird weiterexistieren, weil ein Weltstaat anders als der Weltmarkt nicht in Sichtweite ist. So ergibt sich an dieser Stelle sofort ein politisch-ökonomisch-ökologisches Paradox. Politik erfüllt sich in der Regeneration von Macht, im Setzen von Regeln und Grenzen, in der Beschaffung von Legitimation für politische Intervention, in der Erzeugung und Pflege von Konsens. Sind diese Prinzipien globalisierbar? Wohl kaum, müßte doch das politische Prinzip der Setzung von Grenzen in die Grenzenlosigkeit des globalen Systems wirklich umschlagen. Dabei sind zwei Grenzfälle denkbar. Entweder werden sehr viele Grenzen gezogen, so daß der globale Raum in territorial klein- 
räumliche und machtpolitisch ohnmächtige Einheiten zergliedert wird, oder die Grenzenlosigkeit entsteht dadurch, daß die politische Grenze und die Oberfläche des Globus koinzidieren. Beide Grenzfälle sind mit der Geschichte der modernen Staatenwelt und den institutionellen Regelsystemen der modernen kapitalistischen Welt nicht vereinbar. Dies ist aber das einzige Argument für die ungebrochene Vitalität des Prinzips der Nationalstaatlichkeit. Es ist von grundsätzlich anderem Zuschnitt als jene Entgegnung, die die Globalität der ökologischen Krise und der ökonomischen Kreisläufe (und Krisen) keineswegs für bedeutend genug hält, um konzeptionelle Korrekturen an der nationalstaatlichen Perspektive vorzunehmen (Senghaas 1992) ${ }^{24}$.

Wenn die Nationalstaaten als alleinige Akteure ungeeignet̂ sind, um die globalen ökologischen und ökonomischen Probleme bewältigen zu können, ein globaler Staat aber eine Illusion ist, wächst den intermediären Institutionen und Organisationen einer wenn nicht globalisierten, so doch transnationalisierten Zivilgesellschaft ein doppelter Komplex von Aufgaben im Zuge der internationalen ökologischen Regimebildung zu: Erstens werden Nicht-Regierungsorganisationen unverzichtbare Vermittler und Multiplikatoren des Konsenses innerhalb je nationaler (oder regionaler) Gesellschaften, um radikale und daher zunächst unpopuläre Maßnahmen zur Reduktion der Schadstoffemissionen in die Luft, die Gewässer und die Böden, zur Schonung von Ressourcen, zur Erhaltung der Räume für Arten, deren »ökonomischer Nutzen« nicht kalkuliert werden kann (und darf), überhaupt im gesellschaftlichen Bewußtsein absenken und von den politischen Instanzen erzwingen zu können. Interessen am Schutz der natürlichen Umwelt sind nicht vertikal abgrenzbare Klasseninteressen oder horizontal zuschreibbare disparitäre Interessen partikularer Gruppen. Sie durchziehen jedes Individuum, betreffen vertikale Klassen und horizontale Gruppen gleichermaßen, von den der Klassenlage geschuldeten Ausweichmöglichkeiten vor ökologischer Degradation durch den temporären Einsatz von Geld einmal abgesehen. In diesem Sinne hat Ulrich Beck Recht, wenn er zugespitzt und ironisch feststellt: »Smog ist demokratisch«. Nicht-Regierungsorganisationen ihrerseits sind daher weder klassenspezifisch noch auf Gruppeninteressen festzulegen, sie haben eher anwaltliche Aufgaben.

24 Auch die Theoretiker der $»$ Wettbewerbsfähigkeit « präferieren die nationale Perspektive. Un die Möglichkeit der Herstellung von regionaler und nationaler Wettbewerbsfähigkeit begründen zu können, müssen sie den Weltmarkt als Grenzen setzenden Funktionsraum aus dem Horizont der Analyse eliminieren, um ihn dann aber doch wieder als Referenz für Wettbewerbsfähigkeit und als Absatzmarkt zu benötigen. Siehe etwa die Ausführungen von Messner/Meyer-Stamer 1993. 
Zweitens sind Nicht-Regierungsorganisationen die Bindeglieder internationaler Netzwerke, die von Nationalstaaten nicht geknüpft werden können, da sie sub-etatistisch, unterhalb der »hohen Politik «, wo Souveränität und daher traditionelle Diplomatie eine Rolle spielen, ausgeworfen werden. Sie sind somit gewissermaßen die Konstituentien der internationalen Zivilgesellschaft ${ }^{25}$. »Menschheitsinteressen « (und Menschenrechte sowie Rechte der Völker) jenseits der nationalen Interessen können Nicht-Regierungsorganisationen, für die Souveränität und nationalstaatliche territorial- und funktionsräumliche Macht kein leitendes Prinzip sind, besser artikulieren als nationale Staaten, die sich auf internationale Verhandlungen einlassen. Die internationalen Netzwerke von Nicht-Regierungsorganisationen sind der politisch-förmliche Ausdruck der Globalität der ökologischen Krise ${ }^{26}$ Die Erfahrungen von Rio zeigen auch, daß Nicht-Regierungsorganisationen nicht schon wegen ihres gemeinsamen intermediären Charakters und wegen der politischen Form, in der sie Politik gestalten, auf gleicher Welle kommunizieren könnten. Rowlands stellt zwar »an unprecedented level of cooperation among some members of the NGO community« fest, beschreibt aber auch die Unterschiede zwischen NGO's aus dem Norden und aus dem Süden, im Ausmaß der Professionalität ihrer Aktivitäten, im Grad ihrer Basis-Verankerung, hinsichtlich des Einflusses, den sie auf die Repräsentanten von großen Nationalstaaten oder von internationalen Institutionen auszuüben vermögen (Rowlands 1992, S. 215ff), indem sie zum Beispiel als »Sand im Getriebe« der Konferenzdiplomatie wirken (Ullrich/

25 Glagow (1992) weist darauf hin, daß Nicht-Regierungsorganisationen nicht überhöht werden sollten. Ihre Kraft stammt aus dem Vertrauen, das ihnen entgegengebracht wird. Vertrauen ist aber eine höchst fragile und leicht dahinschwindende Ressource, wenn sie nicht gepflegt wird. Viele Nicht-Regierungsorganisationen hängen am »staatlichen Tropf«, sind also nicht unabhängig. Manche NGO's haben nachgerade kriminelle Energie entwickelt, um an Mittel heranzukommen. Auch sind die Ungleichheiten zwischen nördlichen und südlichen NGO's beträchtlich; darauf verweist auch Rowlands (1992) in seinem Bericht über die NGO's im Verlauf des UNCED-Prozesses. Vgl. auch die Darstellung von Umweltbewegungen in der Entwicklungspolitischen Zusammenarbeit und die Gefahr der »ökozentrischen« und »eurozentrischen« Sichtweise Ullrich/ Kürzinger-Wiemann 1993.

26 Beispiele für internationale NGO-Netzwerke sind etwa der vom BUND geförderte Klimaschutz von unten oder das »Klimabündnis« (Bündnis europäischer Städte mit den indigenen Völkern der Regenwälder zum Ethalt der Erdatmosphäre«) zur Reduktion des Kohlendioxydausstoßes und zur Erhaltung der Regenwälder, die großen international operierenden NGO's wie Greenpeace oder Friends of the Earth. Dazu gehören aber auch der »buisiness council for sustainable development $\ll$, an dem Unternehmer und Unternehmen beteiligt sind und der von Weinzierl in seinem Bericht über die UNCED-Konferenz als »council for sustainable business« apostrophiert wird (Weinzierl 1993: 11). Auch die Gewerkschaften muißten wegen der Bedeutung, die sie als Organisationen der Interessen der Arbeit spielen, Initiativen unternehmen, um sich zur Verteidigung der Umwelt transnational und international zu vernetzen. 
Kürzinger-Wiemann 1993: 168f.). Die Zuschreibung gemeinsamer und möglicherweise einheitlicher Sichtweisen, Interessen, Machtressourcen würde der Vielfalt der NGO's heute nicht mehr gerecht werden.

Ist die internationale Regimebildung mit den Ansätzen der Herausbildung einer trans- bzw. internationalisierten »civil society« ein Schritt zur »sustainability« des globalen Wirtschaftens? Sind so die Zauberlehrlinge der Weltbeherrschung zur Raison zu bringen? Das »okzidentale Modell der Weltbeherrschung«, mit den ungeheuren Potenzen der Industrialisierung vor wenig mehr als 200 Jahren eingeleitet, ist weder im globalen Raum noch für eine mittlere Zeitperspektive (von - sagen wir - einigen Generationen) auf dem Globus zu verallgemeinern, es ist ein exklusives Modell und die Hauptanstrengung bezieht sich heute darauf, die Exklusivität immer wieder gegen die Ansprüche der Gleichheit des Zugriffs auf globale Ressourcen zu verteidigen. Es gibt keine systemimmanente Grenze, an der kapitalistisches Wachstum aufhört und die Expansion stoppt, selbst wenn äußere Grenzen erreicht worden sind, es sei denn die Grenze tritt als Katastrophe in die Alltagswelt. Die Grenze der Belastbarkeit der globalen und regionalen Ökosysteme bleibt der gesellschaftlichen Dynamik in kapitalistischen Marktwirtschaften äußerlich und kann demzufolge auch nur nach extraökonomischen Kriterien markiert werden. Das ist ganz zweifelsfrei ein sozialer $\gg$ Konstruktionsfehler«, der immer deutlicher zu Tage tritt, je näher sich das Modell der Vervollkommnung der intendierten Weltbeherrschung nähert. Folglich ist die Annahme von »Nachhaltigkeit« unter kapitalistischen Bedingungen eine Illusion. Letztlich dürfte nur eine $\gg$ Sonnenstrategie (Scheer 1993) aus dem »fordistischen Fossilismus« heraussteuern.

\section{Literatur}

Aglietta, Michel (1979): A Theory of Capitalist Regulation. The US Experience, London.

Altmann, Jörn (1992): Das Problem des Umweltschutzes im internationalen Handel, in: Entwicklung und Umwelt, Schriften des Vereins für Socialpolitik, Band 215, Berlin, S. 207244.

Altvater, Elmar (1987): Sachzwang Weltmarkt. Verschuldungskrise, blockierte Industrialisierung, ökologische Gefährdung - der Fall Brasilien, Hamburg.

Altvater, Elmar (1991): Die Zukunft des Marktes. Ein Essay über die Regulation von Geld und Natur nach dem Scheitern des »real existierenden Sozialismus «, Münster.

Altvater, Elmar (1991a): Ressourcenkrieg am Golf? Das Öl und die neue moralische Weltordnung, in: PROKLA 82, S. 157-168

Altvater, Elmar (1992): Der Preis des Wohlstands. Umweltplünderung in der neuen Welt(un)ordnung, Münster.

Altvater, Elmar (1993): Die Ökologie der neuen Welt(un)ordnung, in: Nord-Süd aktuell, 1. Quartal 1993, S. 72-84.

Altvater, Elmar/ Mahnkopf, Birgit (1993): Gewerkschaften vor der europäischen Herausforderung, Münster.

Anders, Günther (1980): Die Antiquiertheit des Menschen, München. 
Anderson, Kym/ Blackhurst, Richard (1992): The Greening of World Trade Issues, Hertfordshire.

Anderson, Kym; Blackhurst, Richard. (Ed.) (1993): Regional Integration and the Global Trading System, New York, London etc.

Anderson, Perry (1992): A Zone of Engagement, London/New York.

Arendt, Hannah (1981): Vita Activa oder Vom tätigen Leben, München.

Betz, Karl/ Lüken gen. Claßen, Mathilde/ Schelkle, Waltraud (1993): Übernutzte Umwelt, unterbeschäftigte Arbeit: Systemkrise oder Systemmerkmal? (1), in: Berliner Debatte $I N$ ITIAL, 6/1993, S. 115-126.

Bruckmeier, Karl (1994): Strategien globaler Umweltpolitik, Münster.

Bunker, Stephen (1985): Underdeveloping the Amazon. Extraction, Unequal Exchangc, and the Failure of the Modern State, Urbana/Chicago.

Cipolla, Carlo M. (1985): Die Industrielle Revolution in der Weltgeschichte, in: Cipolla/Borchardt (Hrsg): Europäische Wirtschaftsgeschichte, Band 3, Stuttgart/ New York, S. $1-10$.

Chomsky, Noam (1993): Wirtschaft und Gewalt. Vom Kolonialismus zur neuen Weltordnung, Lüneburg.

Crosby, Alfred (1991): Die Friichte des weissen Mannes, Darmstadt.

Daly, Herman E. (1991): Steady-State Economics, Washington.

Daly, Herman (1994): Die Gefahren des freien Handels, in: Spektrum der Wissenschaft, Januar 1994, S. 40-46.

Debeir, Jean-Claude/ Deléage, Jean-Paul/ Heméry, Daniel (1989): Prometheus auf der Titanic. Geschichte der Energiesysteme, Frankfurt/ New York.

Ely, John (1989): An Ecological Ethic? Left Aristotelian Marxism versus the Aristotelian Right, in: Capitalism, Nature, Socialism, No. 2, Summer 1989, S. 143-156.

French, Hilary F. (1993): Reconciling Trade and the Environment, in: Brown, Lester R., (ed.), State of the World, New York/ London, S. 158-179.

Gellner, Ernest (1991): Nationalismus und Moderne, Berlin.

Georgescu-Roegen, Nicholas (1971): The Entropy Law and the Economic Process, Cambridge/London.

Georgescu-Roegen, Nicholas (1986): The Entropy Law and the Economic Process in Retrospect, in: Eastern Economic Journal, Vol XII, No. 1, 1986, S. 3-25.

Glagow, Manfred (1992): Nicht-Regierungs-Organisationen (NGO) - Neue Hoffnungsträger der internationalen Entwicklungspolitik?, in: Entwicklungspolitische Korrespondenz, 2, 1992, S. 6-8.

Gregory, R.G. (1976): Some Implications of the Growth of the Mineral Sector, in: The Autralian Journal of Agricultural Economics, Vol. 20, No. 2, S. 71-91.

Grundmann, Reiner (1991): The EcologicaI Challenge to Marxism, in: New Left Review 187, S. $103-120$.

Harrod, Roy (1958): The Possibility of Economic Satiety - Use of Economic Growth for Improving the Quality of Education and Leisure, in: Problems of United States Economic Development (Committee for Economic Development, Vol I) New York, S. 207-213.

Hein, Wolfgang (1993): Umweltorientierte Entwicklungspolitik, 2. erw. Aufl., Hamburg.

Heins, Volker (1993): »Survival of the fattest? «. Genetische Ressourcen und globale Biopolitik, in: Peripherie 51/52, S. 69-85.

Hirsch, Fred (1980): Die sozialen Folgen des Wachstums. Eine ökonomische Analyse der Wachstumskrise, Reinbek.

Hirsch, Joachim (1994): Vom fordistischen Sicherheitsstaat zum nationalen Wettbewerbsstaat - Internationale Regulation, Demokratie und »radikaler Reformismus«, in: Das Argument 203, S. 7-22.

Hont, Istvan (1990): Free trade and the economic limits to national policics: a neo-Machiavellian political economy reconsired, in: Dunn, John, (ed.), The economic limits to modern politics, Cambridge/New York, S.41-120.

Hübner, Kurt (1989): Theorie der Regulation. Eine kritische Rekonstruktion eines neuen Ansatzes der Politischen Ökonomie, Berlin. 
Hurtienne, Thomas (1986): Fordismus, Entwicklungstheorie und Dritte Welt, in: Peripherie 22/23, S. 60-110.

Innis, Harold (1956): Essay in Canadian Economic History, Toronto.

Jahn, Thomas; Wehling, Peter (1991): Ökologie von rechts, Frankfurt.

Kennedy, Paul (1993): In Vorbereitung auf das 21. Jahrhundert, Frankfurt.

Keohane, Robert O. (1990): International liberalism reconsidered, in: Dunn, John, (ed.), The economic limits to modern politics, Cambridge/New York, S. 165-194.

Klauss, Jochen (1989): Goethe unterwegs. Eine kulturgeschichtliche Betrachtung, Weimar.

Klauss, Jochen (1990): Alltag im klassischen Weimar, Weimar.

Knieper, Rolf (1991): Nationale Souveränität. Versuch über Ende und Anfang einer Weltord nung, Frankfurt.

Knieper, Rolf (1993): Staat und Nationalstaat. Thesen gegen eine fragwürdige Identität, in: PROKLA, S. 65-71.

Krasner, Stephen D. (1976): State Power and the Structure of International Trade, in: World Politics, No. 28, S. 317-347.

Kulessa, Margareta E. (1992): Free Trade and Protection of the Environment: Is the GATT in Need of Reform?, in: Intereconomics, Vol. 27, July/August 1992, S. 165-173.

Langhammer, Rolf J./Hiemenz, Ulrich (1990): Regional Integration among Developing Countries. Opportunities, Obstacles and Options, Tübingen.

Lipietz, Alain (1993): Berlin, Bagdad, Rio, Münster.

Luhmann, Nikals (1990): Ökologische Kommunikation. Kann die moderne Gesellschaft sich auf ökologische Gefährdungen einstellen? Opladen.

Maddison, Angus (1989): The World Economy in the 20th Century, Paris.

Marx, Karl, Das Kapital in: $M E W$, Band 23.

Massarrat, Mohssen (1993): Endlichkeit der Natur und Überfluss in der Marktökonomie. Schritte zum Gleichgewicht, Marburg.

Messner, Dirk; Meyer-Stamer, Jörg (1993): Die nationale Basis internationaler Wettbewerbsfähigkeit, in: Nord-Süd aktuell, Nr. 1, S. 98-111.

Miller, Lynn H. (1994): Global Order. Values and Power in International Politics, Boulder/San Francisco/Oxford.

Niethammer, Lutz (1990): Posthistoire - Ist die Geschichte zu Ende? Reinbek.

Nitsch, Manfred (1993): Vom Nutzen des systemtheoretischen Ansatzes für die Analyse von Umweltschutz und Entwicklung - mit Beispielen aus dem brasilianischen Amazonasgebiet, in: Sautter, Hermann (Hrsg): Unweltschutz und Entwicklungspolitik, Berlin, S. 235-269.

O'Connor, James (1991): Mord im Orientexpress. Die politische Ökonomie des Golfkriegs, in: PROKLA 84, S. 368-383.

OECD (1991): OECD Environmantal Data, Compendium 1991, Paris.

OECD (1993): Intra-Firm Trade, Paris.

Olson, Mancur (1965): The Logic of Collective Action. Public Goods and the Theory of Groups, Cambridge, Mass.

Ostrom, Elinor (1993): Governing the Commons. The Evolution of Institutions for Collective Action, Cambridge/New York.

Petersmann, Ernst-Ulrich (1992): Umweltsehutz und Welthandelsordnung im GATT-, OECDund EWG-Rahmen, in: Europa-Archiv, 9/92, S. 257-266.

Polany, Karl (1978): The Great Transformation, Frankfurt

Polany, Karl (1979): Ökonomie und Gesellschaft, Frankfurt.

Poulantzas, Nicos (1978): Staatstheorie. Politischer Überbau, Ideologie, Sozialistische Demokratie, Hamburg.

Prigogine, llya; Stengers, Isabelle (1986): Dialog mit der Natur. Neue Wege naturwissenschaftlichen Denkens, 5. Aufl., München.

Prittwitz, Volker von (1990): Das Katastrophenparadox. Elemente einer Theorie der Umweltpolitik, Opladen.

Rigaux, François (1991): Reflexionen über eine neue Weltordnung, in: PROKLA 84, S. 384399. 
Rowlands, Ian H. (1992): The International Politics of Environment and Development: The Post-UNCED Agenda, in: Millenium. Journal of International Studies, Vol. 21, No. 2, S. 209-224.

Ruggie, John Gerard (1993): Territoriality and beyond: problematizing modernity in international relations, in: International Organisation 47, 1, S. 139-174.

Sachs, Wolfgang (1992): Von der Verteilung der Reichtïmer zur Verteilung der Risiken, in: Universitas, Heft 9/92, S. 887-897.

Scharpf, Fritz W. (1987): Sozialdemokratische Krisenpolitik in Europa, Frankfurt.

Scheer, Hermann (1993): Sonnenstrategie. Politik ohne Alternative, München.

Schmitt, Carl (1974): Der Nomos der Erde im Völkerrecht des Jus Publicum Europaeum, Berlin.

Senghaas, Dieter (1992): Weltordnung, aber welche? Weltökonomie und denationalisierte Staatlichkeit in der Perspektive Rolf Kniepers, in: Blätter für deutsche und internationale Politik, S. $1069 \mathrm{ff}$.

Sieferle, Rolf (1989): Die Krise der menschlichen Natur. Zur Geschichte eines Konzepts, Frankfurt.

Simonis, Georg (1993): Der Erdgipfel von Rio - Versuch einer kritischen Verortung, in: Peripherie, Nr. 51/52, S. 12-37.

Stiftung Entwicklung und Frieden (1993): Globale Trends 93/94. Daten zur Weltentwicklung, Frankfurt.

Streeten, Paul (1992): The evolution of development thought: facing up to global interdependence, in: Ekins, Paul; Max-Neef, Manfred, (eds.), Real-Life Economics. Understanding Wealth Creation, London/ New York.

Third World Guide (1993): Third World Guide 93/94. The World as seen by the Third World. Facts, Figures, Opinions, Toronto.

Thompson, William R; Vescera, Lawrence (1992): Growth waves, systemic openness, and protection, in: International Organization, 46, 2, Spring 1992, S. 493-532

Ulrich, Detlev; Kürzinger-Wiemann, Edith (1993): Umweltbewegung und Entwicklungspolitik, in: Hein, Wolfgang (Hrsg.): Umweltorientierte Entwicklungspolitik, Hamburg.

Viner, Jacob (1950): The Customs Union Issue, New York.

Wallerstein, Immanuel (1984): Der historische Kapitalismus, Berlin.

Walzer, Michael (1967): On the Role of Symbolism in Political Thought, in: Political Science Quarterly, June.

Watkins, Mel (1981): The Staple Theory Revisited, in: Melody, William; Salter, Liora; Heyer, Paul, (eds.), Culture, Communication, and Dependency. The Tradition of H. A. Innis, Norwood, S. 53-71.

Weinzierl, Hubert (1993): Der Erdgipfel: Ein Stimmungsbild aus Rio, in: Engelhardt; Weinzierl (Hrsg), Der Erdgipfel. Perspektiven für die Zeit nach Rio, Bonn, S. 1-21.

Weltbank (1993): Weltentwicklungsbericht. Washington.

Whaliey, John (1991): The Interface Between Environmental and Trade Policies, in: The Economic Journal, 1001, March, S. 180-189.

Wissenschaftlicher Beirat der Bundesregierung Globale Umweltveränderungen (1993): Welt im Wandel. Grundstruktur globaler Mensch-Umwelt-Beziehungen, Jahresgutachten 1993, Bonn.

Zarsky, Lyuba (1993): Lessons of Liberalization in Asia. From Structural Adjustment to Sustainable Development, Nautilus Pacific Research, San Diego (Mimeo). 